\title{
LATTICE-ORDERED ALGEBRAS THAT ARE SUBDIRECT PRODUCTS OF VALUATION DOMAINS
}

\author{
MELVIN HENRIKSEN, SUZANNE LARSON, JORGE MARTINEZ, AND R. G. WOODS
}

\begin{abstract}
An $f$-ring (i.e., a lattice-ordered ring that is a subdirect product of totally ordered rings) $A$ is called an $S V$-ring if $A / P$ is a valuation domain for every prime ideal $P$ of $A$. If $M$ is a maximal $\ell$-ideal of $A$, then the rank of $A$ at $M$ is the number of minimal prime ideals of $A$ contained in $M$, rank of $A$ is the sup of the ranks of $A$ at each of its maximal $\ell$-ideals. If the latter is a positive integer, then $A$ is said to have finite rank, and if $A=C(X)$ is the ring of all real-valued continuous functions on a Tychonoff space, the rank of $X$ is defined to be the rank of the $f$-ring $C(X)$, and $X$ is called an $S V$-space if $C(X)$ is an $S V$-ring. $X$ has finite rank $k$ iff $k$ is the maximal number of pairwise disjoint cozero sets with a point common to all of their closures. In general $f$-rings these two concepts are unrelated, but if $A$ is uniformly complete (in particular, if $A=C(X)$ ) then if $A$ is an $S V$-ring then it has finite rank. Showing that this latter holds makes use of the theory of finite-valued lattice-ordered (abelian) groups. These two kinds of rings are investigated with an emphasis on the uniformly complete case. Fairly powerful machinery seems to have to be used, and even then, we do not know if there is a compact space $X$ of finite rank that fails to be an $S V$-space.
\end{abstract}

\section{INTRODUCTION}

By an $f$-ring we mean a lattice-ordered ring which is a subdirect product of totally ordered rings. An $f$-ring $A$ is called an $S V$-ring if $A / P$ is a left valuation domain for each prime ideal $P$ of $A$. ( $D$ is a left valuation domain if it is a ring without proper divisors of zero, and, given any two elements of $D$, one divides the other on the left.) Since any homomorphic image of a left valuation domain (which is a domain) is again a left valuation domain, it follows that $A$ is an SV-ring if and only if $A / P$ is a left valuation domain for each minimal prime ideal of $A$. We point out that there is no reason, in principle, why this concept cannot be regarded without an underlying order; we shall not pursue such an investigation, however.

We shall be dealing with algebras $A$ over the real field $\mathbf{R}$ in the sequel. By an $f$-algebra we mean an $f$-ring which is also a real algebra, in which a positive scalar multiple of a positive element is positive. An $S V$-algebra is understood to be an $f$-algebra which is an SV-ring as well.

In this article all topological spaces will be Tychonoff, that is, they will be endowed with a base of cozero sets. If $X$ is a Tychonoff space, then $C(X)$

Received by the editors January 11, 1993 and, in revised form, September 9, 1993.

1991 Mathematics Subject Classification. Primary 06F15, 06F25, 54C40, 54D99. 
denotes the $f$-ring of all real-valued, continuous functions defined on $X$; it is an $f$-ring under the familiar pointwise operations. If $C(X)$ is an SV-ring we say that $X$ is an $S V$-space. SV-spaces have been studied in [HW1] and [HW2], and SV-rings in [HL]. We shall remind the reader of a number of results from these articles, as warranted in the development of this investigation. However, for additional background information the reader should consult these, as well as $[\mathrm{AF}]$ and $[\mathrm{BKW}]$ for the general theory of lattice-ordered algebraic structures. In this article the most appealing results will probably be the ones for uniformly complete SV-rings. However, we shall also present structure theorems for local SV-rings. In the final sections we shall be concerned with the interaction between the topological structure of a space $X$ and the algebra $C(X)$, in case the latter is an SV-ring.

Recall that an $\ell$-ideal is the kernel of some lattice-preserving ring homomorphism ( $\ell$-homomorphism) between two $f$-rings. By a prime $\ell$-ideal we mean an $\ell$-ideal which is prime as a ring ideal. We will need to consider certain additive $\ell$-subgroups of an $f$-ring. For this reason let us distinguish the notion of prime $\ell$-ideal from that of an $\ell$-prime convex $\ell$-subgroup. Suppose that $G$ is any lattice-ordered group. A subgroup $C$ is convex if whenever $0 \leq a \leq b$ and $b \in C$, then $a \in C$. The subgroup $C$ is called a convex $\ell$-subgroup if it is closed under the lattice operations and is convex. Thus, it is easy to see that an $\ell$-ideal of an $f$-ring is simply a ring ideal which is also a convex $\ell$-subgroup. Now we say that the convex $\ell$-subgroup $N$ of the lattice-ordered group $G$ is $\ell$-prime if $a \wedge b=0$ implies that either $a$ or $b$ belong to $N$. It is well known that a convex $\ell$-subgroup $N$ of $G$ is $\ell$-prime if and only if (1) $G / N$ is totally ordered, or if and only if (2) the set of convex $\ell$-subgroups of $G$ that contain $N$ forms a chain under inclusion. (See 2.4.1 of [BKW].)

While some of our assertions about $\ell$-groups are true for arbitrary ones, the reader should assume that " $\ell$-group" means "abelian $\ell$-group" unless the contrary is stated.

Recall that a ring $A$ is semiprime if the intersection of all the prime ideals of $A$ is $\{0\}$. If $A$ is an $f$-ring then it is semiprime if and only if there are no nonzero nilpotent elements in $A(8.5$, [BKW]). Note that in any $f$-ring, $a \wedge b=0$ means that $a b=0$. The converse holds in semiprime $f$-rings $(9.3 .1$, [BKW]):

(1.0) In a semiprime $f$-ring, $a b=0$ if and only if $a \wedge b=0$.

Other facts we will make use of include:

(1.1) (9.3.1, [BKW]) In a semiprime $f$-ring the minimal prime ideals and the minimal $\ell$-prime convex $\ell$-subgroups coincide.

(1.2) If $A$ is an $f$-ring, then the set $N(A)$ of all nilpotent elements of $A$ coincides with the intersection of the prime ideals of $A$.

(1.3) Every minimal prime ideal of a semiprime $f$-ring is an $\ell$-ideal. A prime ideal $P$ of the semiprime $f$-ring $A$ is minimal if and only if for each $a \in P$ there exists a $b \notin P$ such that $a b=0$. (For lattice-ordered groups there is an analogous characterization of the minimal $\ell$-prime convex $\ell$-subgroups: if $N$ is a prime convex $\ell$-subgroup of $G$ then it is a minimal $\ell$-prime if and only if for each $0 \leq a \in N$ there exists a positive element $b \notin N$ such that $a \wedge b=0$; see [BKW].)

(1.4) Every prime ideal in an $f$-ring with identity is contained in a unique maximal $\ell$-ideal. 
We denote the set of minimal prime ideals of an $f$-ring $A$ by $\operatorname{Min}(A)$. Typically, this set is regarded as a topological space, relative to the so-called hullkernel topology, which is defined in Section 2 below; see [HJ].

The following simple observation for SV-rings is established for a $C(X)$ in [HW1].

Proposition 1.5. Every $\ell$-homomorphic image of an $S V$-ring is an SV-ring.

Proof. Suppose $\theta: A \rightarrow B$ is a surjective $\ell$-homomorphism with kernel $K$, and $A$ is an SV-ring. As was noted in [HL], it suffices to show that $B / Q$ is a left valuation domain for each prime $\ell$-ideal $Q$ of $B$. Every prime $\ell$-ideal $Q$ of $B$ has the form $P / K$ for some prime $\ell$-ideal $P$ of $A$ that contains $K$. Since $A / P$ and $(A / K) /(P / K)$ are isomorphic, the $f$-ring $B / Q$ is an $\ell$-homomorphic image of the left valuation domain, and hence is a left valuation domain. Thus, $B$ is an SV-ring.

Recall from Chapter 3 of [GJ] that a subspace $Y$ of a (Tychonoff) space $X$ is said to be $C^{*}$-embedded in $X$ if the map that sends each bounded $f$ in $C(X)$ to its restriction to $Y$ is a surjective homomorphism, and that every closed subspace of a normal space is $C^{*}$-embedded. Recall also from [HW1] that $C(X)$ is an SV-ring if and only if its subring $C^{*}(X)$ of bounded elements is an SV-ring. Hence we have by Proposition 1.5:

Corollary 1.5.1. Every closed subspace of a normal $S V$-space is an $S V$-space.

We say that an $f$-ring $A$ with identity has bounded inversion if $a \geq 1$ implies that $a$ has a multiplicative inverse. As shown in [HL, 2.7-2.8], $A$ (with identity) has bounded inversion if and only if each maximal left ideal is a (maximal, two-sided) $\ell$-ideal.

Proposition 1.6. Suppose $A$ is an SV-ring with identity element and bounded inversion. If $P$ is a prime $\ell$-ideal and $0 \leq a \leq b$, there exists $a c \in A$ such that $a=c b \bmod P$.

Proof. Suppose $0 \leq a \leq b$ and $P$ is a prime $\ell$-ideal. By hypothesis, $A / P$ is a valuation ring, so either $a=c_{1} b \bmod P$ for some $c_{1} \in A$ or $b=c_{2} a \bmod P$ for some $c_{2} \in A$. In the first case, we are done. In the second case, since $A / P$ is totally ordered and $0 \leq a \leq b$ we may assume that $c_{2} \geq 1$. Since $A$ has bounded inversion, $c_{2}^{-1} \in A$ and $c_{2}^{-1} b=a \bmod P$.

Recall from Chapter 14 in [GJ] that a Tychonoff space such that every finitely generated ideal is principal is called an $F$-space. A space such that its StoneČech compactification $\beta X$ is a union of finitely many closed F-spaces is said to be finitely an F-space.

It is shown in [HW1] that $X$ is an SV-space if and only if $\beta X$ is an SVspace and that if $X$ is finitely an F-space, then $X$ is an SV-space. Whether the converse of this latter question holds remains an open question.

A consequence of one of our main theorems on uniformly complete SV-rings is that if $X$ is finitely an F-space then every maximal ideal of $C(X)$ contains only a finite number of minimal prime ideals. This motivates us to define the notion of rank of a maximal $\ell$-ideal in an $f$-ring, as follows: 
Definitions 1.7. Suppose that $M$ is a maximal $\ell$-ideal of the $f$-ring $A$; let $\operatorname{rk}(A, M)$ denote the number of minimal prime ideals contained in $M$, if the set of all such minimal prime ideals is finite, and $\operatorname{rk}(A, M)=\infty$, otherwise. We then let $\operatorname{rk}(A)$ stand for the supremum of all $\operatorname{rk}(A, M)$. We speak of the rank of a maximal $\ell$-ideal in referring to $\operatorname{rk}(A, M)$, and say that $A$ has finite rank if $\operatorname{rk}(A)$ is finite. If $X$ is a topological space we write $\operatorname{rk}(X)=$ $\operatorname{rk}(C(X))$, and call this number the rank of $X$. For $p \in X$, we write $\operatorname{rk}(X, p)$ for $\operatorname{rk}\left(C(X), M_{p}\right)$, where $M_{p}$ denotes the maximal ideal of all functions which vanish at $p ; \operatorname{rk}(X, p)$ will be called the rank of $p$ in $X$.

For later use, we record companions to Proposition 1.5 and its corollary, the first of which is a result about the behavior of ranks under an $\ell$-homomorphism.

Proposition 1.8. Suppose that $\theta: A \rightarrow B$ is an $\ell$-homomorphism of semiprime $f$-rings with identity and the bounded inversion property, which is onto $B$, having kernel $K$. If $M$ is a maximal ideal of $A$, containing $K$, then $\operatorname{rk}(A, M) \geq$ $\operatorname{rk}(B, \theta(M))$.

Proof. First, recall that if $M$ is a maximal ideal, which contains the kernel of an $\ell$-homomorphism, then its image is again a maximal ideal, and moreover, every maximal ideal of $B$ will arise in this fashion.

If the rank of $M$ is infinite there is nothing to prove. So let us assume that $\operatorname{rk}(A, M)=k<\infty$. This implies that any set of pairwise incomparable prime $\ell$-ideals, contained in $M$, contains at most $k$ members. Next, observe that the minimal prime ideals of $B$ which lie in $\theta(M)$ are in one-to-one correspondence with the prime $\ell$-ideals of $A$, lying in $M$, which are minimal with respect to containing $K$. Thus, $\operatorname{rk}(B, \theta(M)) \leq k$.

Corollary 1.8.1. If $Y$ is a closed subspace of a normal space $X$ and $p \in Y$, then $\operatorname{rk}(Y, p) \leq \operatorname{rk}(X, p)$.

Since a compact subspace is always $C^{*}$-embedded one also gets:

Corollary 1.8.2. Suppose that $Y$ is a compact subspace of the space $X$, and $p \in Y$, then $\operatorname{rk}(Y, p) \leq \operatorname{rk}(X, p)$.

Throughout, $\mathbf{N}$ will denote the discrete space of positive integers. If $A$ is an $f$-ring with identity, we denote by $A^{*}$ the subring $\{a \in A:|a| \leq n \cdot 1$, for some $n \in \mathbf{N}$, which is referred to as the bounded subring of $A$. Clearly $A^{*}$ is a convex $\ell$-subring of $A$.

For any $f$-ring $A$ with identity, let $\operatorname{Max}(A)$ denote the set of all maximal $\ell$ ideals of $A$. If $A$ is a semiprime $f$-ring with identity, having bounded inversion, then, as in the proof of 2.8 in [HL], each maximal ideal of $A$ is an $\ell$-ideal. Thus, for semiprime $f$-rings with identity, having bounded inversion, $\operatorname{Max}(A)$ is, in fact, the set of maximal ideals of $A$.

Suppose that $A$ is a sublattice and subring of the $f$-ring $B$, both with (the same) identity. Consider the map $\theta: \operatorname{Max}(B) \rightarrow \operatorname{Max}(A)$ defined by: $\theta(M)=$ $N$ if $N$ is the maximal $\ell$-ideal of $A$ containing $M \cap A$. Scott Woodward, in his University of Florida dissertation [W], shows for commutative semiprime $f$-rings with bounded inversion, that $\theta$ is a continuous surjection. In particular, for the subring $A^{*}$ of $A$, the map $\theta$ turns out to be a homeomorphism. Let us agree to call an $f$-ring $A$ with identity a bounded $f$-ring, if $A=A^{*}$. Note that if $A$ and $B$ are both bounded $f$-rings satisfying the bounded inversion 
property, and $A$ is an $\ell$-subring of $B$, then $\theta(M)=M \cap A$, for each $M \in$ $\operatorname{Max}(B)$. This is so because if $A$ is bounded and has the bounded inversion property, then $A / M$ is isomorphic to an ordered subfield of $\mathbf{R}$.

Proposition 1.9. Suppose that $A$ is an $\ell$-subring of $B$, both semiprime $f$-rings having the same identity, as well as the bounded inversion property. Let $M \in$ $\operatorname{Max}(B)$, and suppose that $\left|\theta^{-1}(\theta(M))\right| \leq n$; then if $\operatorname{rk}(B, M) \leq k$ it follows that $\operatorname{rk}(A, \theta(M)) \leq n k$.

Proof. Without loss of generality, we may assume that both rings are bounded. It suffices to prove that any set of $\ell$-prime convex $\ell$-subgroups which are contained in $\theta(M)$, and are pairwise incomparable, has at most $n k$ elements.

If $P$ is an $\ell$-prime convex $\ell$-subgroup of $A$, contained in $\theta(M)$, then $P=K \cap A$, for some convex $\ell$-subgroup $K$ of $B$. Evidently, $1 \notin K$, and so $K$ is contained in a convex $\ell$-subgroup $M_{p}$ of $B$ which is maximal with respect to excluding the identity. It is not hard to see, in view of the fact that $B$ is bounded, that $M_{p}$ is, in fact, a maximal ideal of $B$. Now $\theta\left(M_{p}\right)=M_{p} \cap A$ contains $P$, whence $\theta\left(M_{p}\right)=\theta(M)$. This is sufficient to establish the goal stated at the end of the previous paragraph, and the proposition is proved.

As a consequence of Proposition 1.9 we get for compact spaces:

Corollary 1.9.1. Suppose that $X$ is compact, and $f: X \rightarrow Y$ is a continuous surjection. If $p \in X$, and at most $n \in \mathbf{N}$ points map to $f(p)$, then if $\operatorname{rk}(X, p) \leq$ $k, \operatorname{rk}(Y, f(p)) \leq n k$.

Proof. The surjection $f$ induces an embedding $C(f)$ of $C(Y)$ in $C(X)$, with all the conditions assumed in the preceding proposition.

Much of our work will be directed at connections between the SV property and the concept of finite rank for rings and topological properties. In this regard, we shall prove that a uniformly complete SV-algebra has finite rank.

We begin our investigation into the structure of SV-rings with a look at local $f$-rings, since, for semiprime $f$-rings, a great deal can be learned by studying their localizations.

\section{LOCAL SV-RINGS}

If $I$ is an ideal of an $f$-ring $A$, let

$$
O(I)=\{a \in A: a x=0 \text { for some } x \notin I\} .
$$

As is noted in 3.5 of [HL], if $A$ is semiprime, and $M$ is a maximal $\ell$-ideal, then $O(M)$ is the intersection of all of the minimal prime ideals of $A$ that are contained in $M$. An $f$-ring will be called local if it has exactly one maximal $\ell$-ideal. (Local $f$-rings need not be commutative.)

If $M$ is a maximal $\ell$-ideal of a semiprime $f$-ring $A$, then by $1.4, A / O(M)$ is a local $f$-ring. Studying these factor rings provides valuable information about semiprime $f$-rings; especially if $A$ is an $S V$-ring. Our work will be made easier by applying facts about $\ell$-groups (abelian and nonabelian) that appear in [AF], [BKW], or [GlH].

Suppose $g$ is an element of an $\ell$-group $G$. A convex $\ell$-subgroup of $G$ maximal with respect to avoiding $g$ is called a value of $g$. Each nonzero $g$ has a value, and by [AF, Theorem 1.2.10], values are $\ell$-prime convex $\ell$ subgroups. If each element of $G$ has finitely many values, then $G$ is said to 
be finite-valued. An element of $G$ with exactly one value is called special. An element is special if and only if the convex $\ell$-subgroup it generates has a unique maximal convex $\ell$-subgroup. By 2.5 .12 of [BKW] :

(2.1) Every special element of an $\ell$-group is either positive or negative.

If $a$ and $b$ are distinct elements of $G$ and $a \wedge b=0$, then $\{a, b\}$ is called disjoint. If $S$ is a subset of $G$ such that every pair of (distinct) elements of $S$ is disjoint, then $S$ is said to be pairwise disjoint.

As usual, let $G^{+}=\{g \in G: g \geq 0\}$. An element $e$ in $G^{+}$is called indecomposable if it is not the sum of a pair of nonzero disjoint elements. Clearly every special element is indecomposable. We will see in Examples 3.3 and 3.4, that the converse of this latter fails to hold.

We pause to give some examples to illustrate some of these concepts. Before doing so, we need to recall some additional definitions.

An element $e$ of $G^{+}$is called a weak (resp. strong) order unit if $e \wedge x=0$ implies $x=0$ (resp. $G=G(e)$ is the smallest convex subgroup containing $e$ ). It is easy to see that every strong order unit is a weak order unit, and that if $G$ is a semiprime $f$-ring, then $e \in G^{+}$is a weak order unit if and only if it is regular.

$G$ is called archimedean if $a \in A^{+}$and the set of positive integral multiples of $a$ is bounded above together imply that $a=0$.

By a Riesz space $V$ is meant a vector lattice over the real field $\mathbf{R}$; that is, $V$ is an $\ell$-group and a vector space over $\mathbf{R}$ such that if $\lambda \in \mathbf{R}^{+}, x \in G^{+}$, then $\lambda x \in V^{+}$.

If $V$ is an archimedean Riesz space and $e \in V^{+}$is a weak order unit, let $X_{e}(A)$ denote the set of values of $e$. If $a \in A$, let $\operatorname{coz} \hat{a}=\left\{M \in X_{e}(A): a \notin\right.$ $M\}$. As is noted in [HR, Section 2], $\{\operatorname{coz} \hat{a}: a \in A\}$ is a base for a compact (Hausdorff) topology on $X_{e}(A)$ such that $\bigcap\left\{M: M \in X_{e}(A)\right\}=\{0\}$. Indeed, $V$ is isomorphic as a Riesz space to a point-separating Riesz space of extended real-valued continuous functions on $X_{e}(A)$; that is continuous functions that take values in the two-point compactification $\mathbf{R} \cup\{ \pm \infty\}$ of the real line $\mathbf{R}$ which are real-valued on an open dense set. This is called the Yosida representation of $A$ with respect to $e$; for details, see [HR] or [LZ]. We will be concerned only with the case when $e$ is a strong order unit. In this case, $A$ may be regarded as a Riesz subspace of $C\left(X_{e}(A)\right)$ and each $M \in X_{e}(A)$ is maximal; see 3.5 of [HR]. This enables us to conclude that:

Example 2.2. If $X$ is a compact space, then the constant function 1 has only finitely many values in $C(X)$ if and only if $X$ is a finite (discrete) space.

If $f \in C(X)$, the set $\{x \in X: f(x) \neq 0\}$ is called the cozero set of $f$ and is denoted by $\operatorname{coz}(f)$. It is an exercise to verify that:

Example 2.3. If $X$ is compact, then an $f \in C(X)^{+}$is special if and only if its cozero set is a singleton.

Being an indecomposable element in a $C(X)$ does not impose such a severe restriction. If $f=g+h$ and $\{g, h\}$ is disjoint, then $\operatorname{coz}(f)=\operatorname{coz}(g) \cup \operatorname{coz}(h)$ and $\operatorname{coz}(g) \cap \operatorname{coz}(h)=\varnothing$. Thus:

Example 2.4. If $\mathrm{X}$ is compact, then $f \in C(X)$ is indecomposable if and only if $\operatorname{coz}(f)$ is connected. 
The following theorem is the fundamental result on the local structure of an $\ell$-group; see [AF, Theorem 10.10].

Example 2.5 (Conrad). An $\ell$-group $G$ is finite-valued if and only if each $g>0$ in $G$ is a sum of finitely many pairwise disjoint special elements.

Thus, unlike the situation in general, we have:

Corollary 2.6. An element of a finite-valued $\ell$-group is special if and only if it is indecomposable.

An element $g>0$ in $G$ is called basic if the convex $\ell$-subgroup that it generates is totally ordered. The $\ell$-group $G$ is said to have a (finite) basis if there is a (finite) maximal pairwise disjoint subset which consists of basic elements.

If $S \subset G$, let $S^{\perp}=\{g \in G:|g| \wedge|s|=0$ for all $s \in S\}$, and if $s \in S$, let $S^{\perp}=\{s\}^{\perp}$. A subset $P$ of $G$ is called a polar if $P=S^{\perp}$ for some $S \subset G$; equivalently if $P=P^{\perp \perp}$. The set $\mathrm{P}(G)$ of all polars of $G$ is a Boolean algebra under set inclusion. A polar of the form $s^{\perp \perp}$ for some $s \in G$ is called a principal polar, and the subset of all principal polars is denoted by $\mathrm{P}_{r}(G)$. Since $g=|g|^{\perp \perp}$, and if $g, h \in G^{+}$, we have $g^{\perp \perp} \cap h^{\perp \perp}=(g \wedge h)^{\perp \perp}$ and $g^{\perp \perp}+h^{\perp \perp}=(g \vee h)^{\perp \perp}, \mathrm{P}_{r}(G)$ is a sublattice of $\mathrm{P}(G)$.

By the lex-kernel $L(G)$ is meant the convex $\ell$-subgroup generated by those elements of $G$ that fail to be weak order units. Equivalently, the lex-kernel is the convex $\ell$-subgroup generated by all principal polars $a^{\perp \perp}$ where $a^{\perp \perp} \neq G$. An $\ell$-group $G$ is a lex-extension of the convex $\ell$-subgroup $C$ of $G$, if (i) $C$ is $\ell$-prime, and (ii) $g>c$ for all $g>0$ and $g \in G^{+} \backslash C$ and $c \in C$. If $C$ is an $\ell$-subgroup of $G$ and is a lex-extension of $U$ for some $U$ properly contained in $C$, then $C$ is called a lex-subgroup of $G$.

Theorem 5.19 and the Corollary to Theorem 5.22 of $[\mathrm{GlH}]$ give some insight into the concepts of special and basic elements.

Theorem 2.7. An element $a>0$ in $G$ is special if and only if $a^{\perp \perp}$ is a lexsubgroup of $G$.

Theorem 2.8. An element $s>0$ in $G$ is basic if and only if $s^{\perp}$ is the unique minimal $\ell$-prime convex $\ell$-subgroup not containing $s$.

Theorem 2.9. Every basic element of an $\ell$-group is special and every special element of an archimedean $\ell$-group is basic.

Proof. Let $s>0$ be a special element of the $\ell$-group $G$. Then the smallest convex $\ell$-subgroup containing $s$ is a totally ordered group and hence a lexextension of $\{0\}$. By 5.1.8 of [GlH], this implies $s$ is special. The fact that every special element of an archimedean $\ell$-group is basic is shown in 11.1.13 of [BKW].

Some examples that illustrate some of these concepts follow.

Examples 2.10. (a) Let $C(X)$ denote the $f$-ring of continuous real-valued functions on the subspace $X=[0,1] \cup\{2\}$ of $\mathbf{R}$, let $s$ denote the characteristic function of $[0,1]$, and let $t$ denote the characteristic function of $\{2\}$.

(i) By 2.3, $s$ is indecomposable. Clearly, $M_{x}=\{f \in C(X): f(x)=0\}$ is a value of $s$ for each $x \in[0,1]$, so $s$ is not special. Because $s^{\perp}=\{f \in C(X)$ : $f([0,1])=0\}$ is not $\ell$-prime, $s$ fails to be basic by 2.7 . 
(ii) Clearly, $M_{2}=t^{\perp}$ is the unique value of $t$, and the convex $\ell$-group it generates is totally ordered. Hence $t$ is both basic and special.

(b) Let $A=\left\{\frac{p(x)}{q(x)}: p(x), q(x) \in \mathbf{R}[x]\right.$ and $\left.q(0) \neq 0\right\}$ with the usual operations on rational functions. Order $\mathbf{R}[x]$ lexicographically with $1 \gg x \gg x^{2} \gg$ $\ldots$, and let $\frac{p(x)}{q(x)}>0$ if $p(x) q(x)>0$ in $\mathbf{R}[x]$. Observe that $A$ is a totally ordered integral domain that is closed under bounded inversion whose unique maximal ideal is $M=x A$. Let $B=\{(\alpha, \beta) \in A \times A:(\alpha-\beta) \in M\}$. It is shown in [HS] that with the ordering induced by $A \times A, B$ is an $f$-ring whose unique maximal ideal is $M \times M$. Clearly $B$ is closed under bounded inversion.

The element $(1,1)$ of $B$ has $M \times M$ as its only value, so $(1,1)$ is special. Because $(1,1)^{\perp}=\{(0,0)\}$ is not $\ell$-prime, it fails to be basic by 2.7 .

It is not difficult to verify that an element of $B$ has at most two values. So the converse of 2.9 fails to hold even for semiprime $f$-rings closed under bounded inversion that are finite-valued.

The following result is extracted from Theorem 5.3.4 of [GlH] and Theorem 2.5 .

Theorem 2.11. For $G$ an $\ell$-group, the following are equivalent:

(a) $G$ has a finite basis.

(b) Each disjoint subset of $G$ is finite.

(c) $G$ has only finitely many minimal $\ell$-prime convex $\ell$-subgroups.

(d) $\mathrm{P}_{r}(G)$ is finite.

(e) Each minimal $\ell$-prime convex $\ell$-subgroup is in $\mathrm{P}_{r}(G)$.

Moreover, any of these implies that $G$ is finite-valued. If $G$ has a basis of $k$ elements, then there is a disjoint subset of $G$ containing $k$ elements and no disjoint subset containing $k+1$ nonzero elements.

The next proposition records some facts about $f$-rings that will be needed below; see [BKW, Chapter 8] and [H]. An $f$-ring is said to be square root closed if the equation $x^{2}=a$ has a solution for each $a \geq 0$.

Proposition 2.12. Suppose $A$ is a semiprime f-ring. Then:

(a) Each of its $\ell$-subrings is a semiprime $f$-ring.

(b) Every polar in $A$ is an $\ell$-ideal.

(c) Every sum of $\ell$-ideals is an $\ell$-ideal.

(d) If $A$ is square root closed, then every sum of semiprime $\ell$-ideals is semiprime.

Lemma 2.13. If $a$ is a special element of a semiprime $f$-ring $A$, then $L\left(a^{\perp \perp}\right)+$ $a^{\perp}$ and $a^{\perp \perp}+a^{\perp}$ are prime $\ell$-ideals of $A$.

Proof. By Proposition 2.12(a,b,c), $L\left(a^{\perp \perp}\right)$ is an $\ell$-ideal of the semiprime $f$-ring $a^{\perp \perp}$. Since $A$ is square root closed and $a^{\perp \perp}$ (resp. $\left.L\left(a^{\perp \perp}\right)\right)$ is semiprime, if $x \in\left(a^{\perp \perp}\right)^{+}$(resp. $\left.x \in L\left(a^{\perp \perp}\right)^{+}\right)$, then so does $\sqrt{x}$. Thus if $x \in L\left(a^{\perp \perp}\right)^{+}$ and $y \in A$, then $y x=(y \sqrt{x}) \sqrt{x} \in L\left(a^{\perp \perp}\right)$, and it follows that $L\left(a^{\perp \perp}\right)$ is a left $\ell$-ideal of $A$. Similarly it is a right $\ell$-ideal of $A$ and hence is an $\ell$-ideal of $A$. By Proposition 2.12(c), $I=L\left(a^{\perp \perp}\right)+a^{\perp}$ is an $\ell$-ideal of $A$. By a simpler application of Proposition 2.12, so is $J=a^{\perp \perp}+a^{\perp}$.

Since $a$ is special, $a^{\perp \perp}$ is a lex-subgroup of $A$. Thus, by 7.1.9 of [BKW], an element $g \in A^{+}$fails to be in $a^{\perp \perp}+a^{\perp}=J$ if and only if $g>a^{\perp \perp}$. It 
follows that if $g, h$ are not in $J$, then neither is $g \wedge h$. It follows that $J$ is a $\ell$-prime convex $\ell$-subgroup of $A$. Since $J$ contains a minimal $\ell$-prime convex $\ell$-subgroup, and minimal $\ell$-prime convex $\ell$-subgroups of semiprime $f$-rings are minimal prime ideals, $J$ contains a prime ideal. Now, polars in an $f$-ring are semiprime ideals, so by $2.12(\mathrm{~d}), J$ is semiprime . Because a semiprime $\ell$-ideal that contains a prime $\ell$-ideal is prime, $J$ is a prime $\ell$-ideal of $A$. (See $[\mathrm{H}]$.)

By 1.1 .5 in $[\mathrm{GlH}]$, to show that the convex $\ell$-subgroup $I$ is $\ell$-prime, it suffices to show that $g \wedge h=0$ for $g, h \in A$ implies $g$ or $h$ is in $I$. Now $L\left(a^{\perp \perp}\right)$ is $\ell$-prime in $a^{\perp \perp}$, so:

$I$ is $\ell$-prime as a convex $\ell$-subgroup of $J$.

Now suppose $g \wedge h=0$ for $g, h \in A$. Since $J$ is prime, one of $g, h$ is in $J$; say $g \in J$. If $h \in J$, then one of $g, h$ is in $I$ by $(*)$. Otherwise, by 7.1.9 of [BKW], $h>a^{\perp \perp}$, so $0=g \wedge h \geq g \wedge a \geq 0$ and we have $g \in a^{\perp} \subset I$. Thus $I$ is an $\ell$-prime convex $\ell$-subgroup. Then, as with $J, I$ contains a prime ideal. Since $L\left(a^{\perp \perp}\right)$ is semiprime and polars in an $f$-ring are also semiprime, 2.12(d) implies $I$ is semiprime. Because a semiprime $\ell$-ideal that contains a prime $\ell$-ideal is prime, $I$ is a prime $\ell$-ideal of $A$. (See $[\mathrm{H}]$.) This completes the proof of the lemma.

We are now ready to state and prove the main result of this section.

Theorem 2.14. Suppose $A$ is a local semiprime $f$-ring of finite rank with identity element and bounded inversion that is square root closed. Then $A$ is an $S V$-ring if and only if whenever $0 \leq a \leq b$, and $b$ is special, there is an $x \in A$ such that $a=x b$.

Proof. Note first that by Theorem $2.11, A$ is finite-valued.

(Sufficiency) Suppose $0 \leq a \leq b$ and $P$ is a prime ideal of $A$. Since $A$ is finite-valued, there are nonzero pairwise disjoint special elements $a_{i}$ for $1 \leq$ $i \leq k$ such that $a=a_{1}+\cdots+a_{k}$. Since $P$ is prime and the $a_{i}$ are pairwise disjoint, there is a unique $j$ such that $a+P=a_{j}+P$. By assumption, there is an $x \in A$ such that $a_{j}=x b$. Hence $a-x b \in P$.

(Necessity) We will prove first that:

( $\dagger$ ) If $|x| \leq m b$ for some positive integer $m$ and special element $b \in A$, then there are $c, d \in A$ such that $x-c b=d$, where $d^{\perp \perp} \subset x^{\perp \perp}$ and $|x| \leq n b$ for some positive integer $n$.

To establish $(\dagger)$, note first that since $x$ is a difference of positive elements, there are by Theorem 2.5 nonzero pairwise disjoint special elements $x_{i}$ such that $x=x_{1}+\cdots+x_{k}$, which by 2.1 are either positive or negative.

Let $y=x_{i}$ for some fixed $i$. By Lemma 2.13, $I=L\left(y^{\perp \perp}\right)+y^{\perp}$ is a prime $\ell$ ideal. Since $A$ is an SV-ring, there are, by Proposition 1.6, $c \in A, d \in L\left(y^{\perp \perp}\right)$, and $d^{\prime} \in y^{\perp}$ such that $|y|-c b=d+d^{\prime}$. We may assume $0 \leq c \leq m$ by replacing $c$ by $(0 \vee c) \wedge m$, while still having $|y|-c b \in I$. We show first that:

$$
\text { there is a } c^{*} \in A \text { such that }|y|-c^{*} b \in L\left(y^{\perp \perp}\right) \text {. }
$$

We consider two cases: (i) $y^{\perp \perp}=b^{\perp \perp}$ and (ii) $y^{\perp \perp} \neq b^{\perp \perp}$. If (i) holds, then $|y|-c b-d=d^{\prime} \in y^{\perp} \cap y^{\perp \perp}=\{0\}$, so $(\sharp)$ holds in this case.

If (ii) holds, note first that $J=y^{\perp \perp}+y^{\perp}$ is a prime $\ell$-ideal by Lemma 2.13 . Since $b$ is special, it is indecomposable. If $b$ were in $J$, then it would be in $y^{\perp}$ or in $y^{\perp \perp}$. The fact that $|y| \leq m b$ implies $y \in b^{\perp \perp}$. If $b \in y^{\perp \perp}$, then 
$b^{\perp \perp}=y^{\perp \perp}$, contrary to (ii). If $b \in y^{\perp}$, then $y=0$ because $|y| \leq m b$ and $A$ is semiprime; whence $b=0$. Thus, $b \notin J$. Now $c b=|y|-d-d^{\prime}$ is in the prime ideal $J$, so $c \in J$ and there are $c^{\prime \prime} \in y^{\perp \perp}$ and $c^{\prime} \in y^{\perp}$ with $c=c^{\prime \prime}+c^{\prime}$. Then $|y|-c^{\prime \prime} b-d=c^{\prime} b+d^{\prime} \in y^{\perp} \cap y^{\perp \perp}=\{0\}$. So $(\sharp)$ holds in this case as well.

Resuming the earlier notation, by $(\sharp)$, there are $c_{i} \in A$ and $d_{i} \in L\left(x_{i}^{\perp \perp}\right)$ such that $\left|x_{i}\right|-c_{i} b=d_{i}$ for $1 \leq i \leq k$. Let $e_{i}=1$ if $x_{i}>0$ and $e_{i}=-1$ if $x_{i}<0$, let $c=e_{1} c_{1}+\cdots+e_{k} c_{k}$, and let $d=e_{1} d_{1}+\cdots+e_{k} d_{k}$. Then $x-c b=d$. Since $|x| \leq m b$ and $0 \leq c_{i} \leq m$ for each $i, \quad\left|d_{i}\right| \leq 2 m b$ and $|d| \leq 2 m k b$.

We show next that:

$$
d^{\perp \perp} \text { is contained properly in } x^{\perp \perp} \text {. }
$$

Again, let $y=x_{i}$ for some fixed $i$. By $2.7, L\left(y^{\perp \perp}\right)$ is contained properly in $y^{\perp \perp}=S$. For any $g \in S$, let $g \stackrel{\perp}{\perp}$ denote the principal polar of $g$ with respect to the subring $S$. Now $d \in L\left(y^{\perp \perp}\right)$, so $d=f_{1}+\cdots+f_{t}$ for some $f_{1}, \ldots, f_{t} \in S^{+}$, where each $f_{j} \in\left(g_{j}\right)_{S}^{\perp \perp}$ for some $g_{j}$ with $\left(g_{j}\right)_{S}^{\perp \perp}$ contained properly in $S$. So $d^{\perp \perp} \subseteq d_{S}^{\perp \perp} \subseteq \bigvee_{j}\left\{\left(f_{j}\right)_{S}^{\perp \perp}\right\} \subseteq \bigvee_{j}\left\{\left(g_{j}\right)_{S}^{\perp \perp}\right\} \subseteq L\left(y^{\perp \perp}\right) \subset S$, where the last containment is proper. Because the $x_{i}$ are pairwise disjoint, $x_{i}^{\perp \perp} \cap x_{j}^{\perp \perp}=\{0\}$ if $i \neq j$. It follows that $d^{\perp \perp} \subset \bigvee_{i}\left\{d_{i}^{\perp \perp}\right\}$, which is contained properly in $\bigvee_{i}\left\{x_{i}^{\perp \perp}\right\}=x^{\perp \perp}$. Hence $(\sharp \sharp)$ holds, and from this it is an exercise to see that $(t)$ holds.

Suppose $0 \leq a \leq b$ and $b$ is special. By $(\dagger)$, there are $c_{0}, d_{1} \in A$ such that $a-c_{0} b=d_{1}$ with $d_{1}^{\perp \perp}$ contained properly in $a^{\perp \perp}$ and $\left|d_{1}\right| \leq n b$ for some positive integer $n$. Repeated applications of $(\dagger)$ yield sequences $c_{i}, d_{i} \in A$ such that $d_{i}-c_{i} b=d_{i+1}$ with $d_{i+1}^{\perp \perp}$ contained properly in $d_{i}^{\perp \perp}$ for $i \geq 1$. Then $a^{\perp \perp} \supset d_{1}^{\perp \perp} \supset d_{2}^{\perp \perp} \supset \cdots$ is a descending chain of principal polars. By Theorem 2.11, there are only finitely many elements in $\mathrm{P}_{r}(A)$, so $d_{p+1}=0$ for some $p$ and $a=\left(c_{0}+\cdots+c_{p}\right) b$. This completes the proof of the theorem.

Suppose $A$ is a reduced ring (i.e. a ring whose only nilpotent element is 0 ) with identity. If every finitely generated left ideal of $A$ is principal, then $A$ is called a left Bezout ring, and if this conclusion holds for regular left ideals, then $A$ is said to be a left quasi-Bezout ring. Let $z(A)$ denote the set of zero-divisors of $A$. Note that $z(A)$ is completely prime (i.e., $A / z(A)$ has no proper divisors of zero) if $z(A)$ is an ideal of $A$. We will call $A$ a strong $S V$-ring if $z(A)$ is an ideal of $A$ and $A / z(A)$ is a left valuation domain.

Proposition 2.15. Suppose $A$ is a semiprime local $f$-ring with identity that is closed under bounded inversion.

(a) If $A$ is left quasi-Bezout, then $z(A)$ is a prime $\ell$-ideal and $A$ is a strong SV-ring.

(b) If $z(A)$ is a minimal prime ideal, then $z(A)=\{0\}$.

(c) If $A$ is left quasi-Bezout and $\operatorname{Min}(A)$ is compact, then $A$ is a left valuation domain.

Proof. (a) We begin by showing that $z(A)$ is an ideal of $A$. Otherwise, there are $x, y \in z(A)$ such that $x+y$ is regular. Then the left ideal generated by $\{x, y, x+y\}$ is principal with regular generator $d=s x+t y$ for some $s, t \in A$. There are $x_{1}, y_{1} \in A$ such that $x=x_{1} d$ and $y=y_{1} d$, so $\left(s x_{1}+t y_{1}\right) d=d$. 
Since $d$ is regular, this yields:

$$
s x_{1}+t y_{1}=1
$$

Let $M$ denote a left ideal of $A$ that contains $s$ and is maximal with respect to not containing $t$. Then $M$ is a maximal left ideal of $A$. For if $w \notin M$, then the smallest left ideal of $A$ that contains $M$ and $w$ contains $t$, and hence must be all of $A$ by $(*)$. Since $A$ is closed under bounded inversion, the maximal left ideal $M$ is an $\ell$-ideal and hence is a maximal ideal by 2.8 in [HL] and the fact that maximal left $\ell$-ideals in an $f$-ring are (two-sided) ideals. Similarly, any left ideal $M^{\prime}$ that contains $t$ and is maximal with respect to not containing $s$ is a maximal ideal of $A$. Clearly, $M \neq M^{\prime}$, so $A$ fails to be a local ring. This contradiction shows that $z(A)$ is an ideal. Because a product of regular elements is regular, $z(A)$ is prime, and because $0 \leq a \leq b \in z(A)$ implies $a \in z(A)$, the latter is also an $\ell$-ideal.

(b) Suppose $a \in z(A)$. Since $A$ is reduced and $z(A)$ is a minimal prime ideal, there is by 2.4 of $[\mathrm{K}]$ a $b \notin z(A)$ such that $a b=0$. Then $a=0$ because $b$ is regular.

Before proving (c), we pause to discuss the hull-kernel topology on $\operatorname{Min}(A)$ for $A$ reduced; for more details, see [HJ], [HK], [K], or [TN]. For $S \subseteq A$, let $h(S)=\{P \in \operatorname{Min}(A): S \subseteq P\}$, let $h(\{s\})=h(s)$ if $s \in A$, and let $h^{c}(S)=\operatorname{Min}(A) \backslash h(S)$. The hull-kernel topology on $\operatorname{Min}(A)$ is the one generated by $\left\{h^{c}(a): a \in A\right\}$. It has the latter as a base and $h^{c}(a)=h\left(a^{\perp}\right)$ whenever $a \in A$. It follows that $\left\{h(a), h\left(a^{\perp}\right)\right\}$ is a pair of disjoint clopen sets whose union is $\operatorname{Min}(A)$, whence $\operatorname{Min}(A)$ is a zero-dimensional Hausdorff space. The following lemma can be inferred from results in $[\mathrm{HJ}]$ or $[\mathrm{TN}]$, but it seems easier to prove it directly.

Lemma 2.16. If $A$ is a semiprime $f$-ring, $\operatorname{Min}(A)$ is compact, and $P \subseteq z(A)$ is a prime ideal, then $P$ is minimal.

Proof. Clearly, $h(P)=\bigcap\{h(p): p \in P\}$. Since each element of $P$ is in $z(A)$, each $h(p)$ is nonempty. Because $A$ is a semiprime $f$-ring, $h\left(p_{1}\right) \cap h\left(p_{2}\right) \cap \cdots \cap$ $h\left(p_{n}\right)=h\left(\left|p_{1}\right|+\left|p_{2}\right|+\cdots+\left|p_{n}\right|\right)$ for any finite subset $\left\{p_{1}, \ldots, p_{n}\right\}$ of $P$. Now $h(P)$ is compact since it is a closed subspace of the compact space $\operatorname{Min}(A)$, and $\{h(p): p \in P\}$ has the finite intersection property. So $h(P)$ is nonempty. Since $P$ is prime, it follows that $P$ is minimal.

Proof of $2.15(\mathrm{c})$. By the last lemma, $z(A)$ is a minimal prime ideal, so by $2.15(\mathrm{~b}), z(A)=\{0\}$. Thus $A$ is a left valuation domain by $2.15(\mathrm{a})$.

For the balance of this section, we concern ourselves with commutative rings with identity. Recall that the classical ring of quotients $q_{c}(A)$ of a commutative ring $A$ is $\left\{\frac{a}{d}: a, d \in A, d \notin z(A)\right\}$ with the usual ring operations. Then $A$ is a subring of $q_{c}(A)$ under the embedding $a \rightarrow \frac{a d}{d}$ for any (fixed) regular $d \in A$. A fractional ideal of $A$ is a (left) submodule of the $A$-module $q_{c}(A)$ for which there is a regular $d \in A$ such that $d I \subseteq A$. A fractional ideal $I$ is invertible if there is a fractional ideal $J$ such that $I J=A$. If every finitely generated regular ideal of $A$ is invertible, then $A$ is called a Prüfer ring. Next we show that:

Lemma 2.17. If $A$ is a local commutative ring with identity, then $A$ is a Prüfer ring if and only if $A$ is (left) quasi-Bezout. 
Proof. Let $I$ denote a finitely generated regular ideal of the local ring $A$. If $A$ is Prüfer, then since $I$ is invertible and $A$ is local, $I$ is principal by 7.5 of Chapter 1 of [G]. So $A$ is quasi-Bezout. Conversely, if $A$ is quasi-Bezout, then $I$ is principal and $I=(d)$ for some regular element $d$. Thus $I$ is invertible in $q_{c}(A)$. Hence $A$ is Prüfer.

Corollary 2.18. Suppose $A$ is a commutative semiprime local $f$-ring with identity and bounded inversion. If $A$ is Prüfer, then $A$ is a strong $S V$-ring. If, in addition $\operatorname{Min}(A)$ is compact, then $A$ is a valuation domain.

In case $A$ is commutative, Theorem 2.15(a) and Lemma 2.17 appear in [MW].

\section{SEMIPRIME $f$-RINGS WHICH HAVE FINITE RANK}

We now recall some terminology and notation about $C(X)$, for a topological space $X$. If $f \in C(X), Z(f)=\{x \in X: f(x)=0\}$ and $\operatorname{coz}(f)$ denotes the complement of $Z(f)$; these are the zero set and cozero set of $f$, respectively. Observe that $f^{\perp}=\{g \in C(X): g(\operatorname{cl}(\operatorname{coz}(f))=0\}$. Also, $f$ is regular if and only $\operatorname{coz}(f)$ is dense in $X$.

It is shown in [HW1] that $X$ is an SV-space if and only if its Stone-Čech compactification $\beta X$ is an SV-space. A consequence of Proposition 3.2 below is that $X$ has finite rank if and only if $\beta X$ has finite rank. So there is no loss of generality in restricting attention to compact spaces.

Suppose $X$ is a compact topological space. If $p \in X$ then $M_{p}=\{f \in C(X)$ : $p \in Z(f)\}$ is a maximal ideal of $C(X)$. According to the Gel'fand-Kolmogorov Theorem (7.3 in [GJ]) the assignment $p \rightarrow M_{p}$ is a homeomorphism from $X$ onto $\operatorname{Max}(C(X))$. We denote

$$
O\left(M_{p}\right)=O_{p}=\{f \in C(X): Z(f) \text { is a neighborhood of } p\} .
$$

To say that $f \in C(X) \backslash O_{p}$ is to say that $p \in \operatorname{cl}(\operatorname{coz}(f))$. Next, we summarize some of the results of Theorem 2.11 for $C(X)$.

Proposition 3.1. Let $X$ be a compact space. Then $p \in X$ has rank $k(<\infty)$ if and only if there is a family of $k$ pairwise disjoint cozero sets with $p$ in each of the closures, but no larger family of pairwise disjoint cozero sets with this feature.

Note: In Corollary 1.8.1 it was mentioned that the rank of a point $p$ lying in a closed subspace $Y$ of the compact space $X$, has rank in $Y$ which cannot exceed its rank in $X$. However, it is a consequence of this proposition that if $p \in \operatorname{int}_{X}(Y)$, then $\operatorname{rk}(Y, p)=\operatorname{rk}(X, p)$.

We have already mentioned that $X$ is an SV-space if and only if $\beta X$ is one. More generally, as shown in Theorem 3.8 of [HL], if $A$ is a semiprime $f$-ring with identity, then $A$ is an SV-ring if and only if $A^{*}$ is one and $A$ has bounded inversion. There are examples (3.9 in [HL]), which show that, without the bounded inversion property on $A, A^{*}$ can be an SV-ring while $A$ is not.

Suppose that $G$ is a lattice-subgroup of the $\ell$-group $H$. We say that $G$ is rigidly embedded in $H$, or that $G$ is a rigid subgroup of $H$, if for each $h \in H$ there is a $g \in G$ so that $h^{\perp \perp}=g^{\perp \perp}$ (where the polars are computed in $H$ ). This concept was introduced in [CM], where it was shown that if $G$ is rigidly embedded in $H$ then the contraction map $N \rightarrow N \cap G$ is a homeomorphism between the two spaces of minimal $\ell$-prime convex $\ell$-subgroups. 
Let $B$ be an $f$-subring rigidly embedded in a semiprime $f$-ring $A$. It is shown in 3.7 of [HL] that the contraction map $P \rightarrow P \cap B$ is a bijection from $\operatorname{Min}(A)$ onto Min $(B)$. Part (2) of the following proposition is the finite rank analogue of 3.8 in [HL].

Proposition 3.2. Let $A$ be a semiprime $f$-ring.

(1) Suppose that $B$ is a rigidly embedded $f$-subring of $A$. Then if $B$ has finite rank, so does $A$.

(2) Suppose that $A$ has an identity element. Then $\operatorname{rk}(A)=\operatorname{rk}\left(A^{*}\right)$. In particular, $A$ has finite rank if and only if $A^{*}$ does.

Proof. Suppose that $\operatorname{rk}(B)=k(<\infty)$. We will show that $\operatorname{rk}(A) \leq \operatorname{rk}(B)$. Suppose not. Then there is a maximal ideal $M$ of $A$ and distinct minimal prime ideals $P_{1}, P_{2}, \ldots, P_{k+1}$ of $A$, with each $P_{i} \subseteq M$. Let $N$ be the unique maximal $\ell$-ideal of $B$ which contains $M \cap B$. For each $i$, then $P_{i} \cap B \subseteq M \cap B \subseteq$ $N$. Since the contraction map $P \rightarrow P \cap B$ is a bijection from $\operatorname{Min}(A)$ onto Min $(B)$, the $P_{i} \cap B$ are distinct minimal prime ideals of $B$ contained in $N$. Hence $\operatorname{rk}(B, N)>k$, a contradiction and the proof of (1) is complete.

Next suppose that $A$ has an identity element. It is easy to see that $A^{*}$ is rigid in $A$, since for any $a \in A, a^{\perp \perp}=((-1 \vee a) \wedge 1)^{\perp \perp}$. We will show that $\operatorname{rk}(A) \geq \operatorname{rk}\left(A^{*}\right)$. Let $M$ be a maximal $\ell$-ideal in $A^{*}$ and suppose $\left\{P_{i}\right\}$ is a collection of distinct minimal prime ideals of $A^{*}$, each contained in $M$. Since the contraction map $P \rightarrow P \cap A^{*}$ is a bijection from $\operatorname{Min}(A)$ onto $\operatorname{Min}\left(A^{*}\right)$, there is for each $i$, a minimal prime ideal $P_{i}^{\prime}$ of $A$ such that $P_{i}^{\prime} \cap A^{*}=P_{i}$, and the $P_{i}^{\prime}$ are distinct. Now all of the $P_{i}^{\prime}$ are contained in the same maximal $\ell$-ideal. For if not, there is some $\alpha, \beta$ such that $P_{\alpha}^{\prime}$ and $P_{\beta}^{\prime}$ are not contained in the same maximal ideal. So $P_{\alpha}^{\prime}+P_{\beta}^{\prime}=A$ and $1 \leq p_{\alpha}+p_{\beta}$ for some positive elements $p_{\alpha} \in P_{\alpha}^{\prime}, p_{\beta} \in P_{\beta}^{\prime}$. This implies $1 \leq\left(p_{\alpha} \wedge 1\right)+\left(p_{\beta} \wedge 1\right)$. Since $\left(p_{\alpha} \wedge 1\right)+\left(p_{\beta} \wedge 1\right) \in\left(P_{\alpha}^{\prime} \cap A^{*}\right)+\left(P_{\beta}^{\prime} \cap A^{*}\right)=P_{\alpha}+P_{\beta}$ and $P_{\alpha}+P_{\beta}$ is an $\ell$-ideal of $A^{*}, P_{\alpha}+P_{\beta}=A^{*}$. But this contradicts the fact that $P_{\alpha}+P_{\beta} \subseteq M \neq A^{*}$. So all of the $P_{i}^{\prime}$ are contained in the same maximal $\ell$-ideal, say $M^{\prime}$. Hence $\operatorname{rk}\left(A, M^{\prime}\right) \geq \operatorname{rk}\left(A^{*}, M\right)$.

We have shown that for any maximal $\ell$-ideal $M$ in $A^{*}$ there is a maximal $\ell$ ideal $M^{\prime}$ in $A$ such that $\operatorname{rk}\left(A, M^{\prime}\right) \geq \operatorname{rk}\left(A^{*}, M\right)$. Therefore $\operatorname{rk}(A) \geq \operatorname{rk}\left(A^{*}\right)$.

If $\operatorname{rk}\left(A^{*}\right)=\infty$, then we are done. If $\operatorname{rk}\left(A^{*}\right)$ is finite, then $\operatorname{rk}(A) \leq \operatorname{rk}\left(A^{*}\right)$ follows from (1).

Note that if $A=C(X)$ for any topological space $X$, then $A^{*}=C^{*}(X)$ is isomorphic to $C(\beta X)$ by 6.6 of [GJ]. So Proposition 3.2(2) implies that $X$ has finite rank if and only if $\beta X$ has finite rank.

The converse of Proposition 3.2(1) is false as is shown in the next example. In it and others that follow, some details are left to the reader.

Example 3.3. Let $\alpha \omega$ denote the one-point compactification of the countable discrete space $\omega$. Then $C(\alpha \omega)$ is rigidly embedded in $C(\omega)$. However, while $C(\omega)$ has rank 1 , the point at $\infty$ in $\alpha \omega$ has infinite rank.

The SV analogues to Proposition 3.2(1) and to its converse are both false, as the following two examples will demonstrate. The first example is of an $f$ subring $B$ rigidly embedded in an $f$-ring $A$ where $A$ is an SV-ring while $B$ 
is not. Note that if $A$ and $B$ are any two totally ordered domains with $B$ a subring of $A$, then $B$ is necessarily rigidly embedded in $A$.

Example 3.4. Let $B$ be any totally ordered commutative domain with bounded inversion, which is not a valuation domain, and $A=q_{c} B$ be its field of fractions. For example, $B$ could be constructed as follows: Consider the polynomial ring $\mathbf{R}[x, y]$ in two variables, lexicographically ordered, so that $1 \gg x \gg x^{2} \gg$ $\cdots \gg y \gg y^{2} \cdots$. Let $B$ be the subring of the field $\mathbf{R}(x, y)$ of rational functions consisting of all fractions $p(x, y) / q(x, y)$ such that $q(0,0) \neq 0$. Give $B$ a total ordering by restricting the natural extension of the order on $\mathbf{R}[x, y]$ to $\mathbf{R}(x, y)$. Then $B$ is rigidly embedded in $A$, and $A=q_{c} B$ is, clearly, a valuation domain while $B$ is not an SV-ring.

The next example is of an $f$-subring $B$ rigidly embedded in an $f$-ring $A$ where $B$ is an SV-ring, but $A$ is not.

Example 3.5. Again consider the polynomial ring $\mathbf{R}[x, y]$ in two variables, lexicographically ordered, so that $1 \gg x \gg x^{2} \gg \cdots \gg y \gg y^{2} \cdots$. Let $A$ be the subring of the field $\mathbf{R}(x, y)$ of rational functions consisting of all fractions $p(x, y) / q(x, y)$ such that $q(0,0) \neq 0$. Give $A$ a total ordering by restricting the natural extension of the order on $\mathbf{R}[x, y]$ to $\mathbf{R}(x, y)$. Now let $B$ be the subring $\{r / 1: r \in \mathbf{R}\}$. Then $B$ is rigidly embedded in $A$, and $B$ is a valuation domain. However, $A$ is not an SV-ring, since it is not a valuation domain: $0<y<x$ and yet there is no $w$ in $A$ such that $y=w x$.

We close this section by showing that in the class of semiprime $f$-rings with bounded inversion, neither having finite rank nor being an SV-ring imply the other. First we give an example of an archimedean semiprime $f$-ring of finite rank with bounded inversion that is not an SV-ring.

Example 3.6. Let $X=\{(0,0)\} \cup\left\{\left(1 / n, 1 / n^{n}\right): n \in \mathbf{N}\right\}$ with the topology inherited from $\mathbf{R}^{2}$. Let

$A=\left\{f \in C(X):\right.$ there exists an $n_{0}$ such that

$$
f\left(1 / n, 1 / n^{n}\right)=p\left(1 / n, 1 / n^{n}\right) / q\left(1 / n, 1 / n^{n}\right),
$$

for all $n \geq n_{0}$, for some $p, q \in \mathbf{R}[x, y]$, with $\left.q(0,0) \neq 0\right\}$.

It is easy to verify that $A$ is a sub-f-ring of $C(X)$ and hence is semiprime and archimedean. Note that the ideal $Q$ of all elements of $A$ which are "eventually zero" is the unique minimal prime ideal of $A$ which is not also maximal. Thus, $A$ has rank one, but if we define $f$ and $g$ by $f(a, b)=b$ and $g(a, b)=a$, for all $(a, b) \in X$, then $0<f<g$, and yet there is no $w \in A$ such that $f=w g \bmod Q$. This says that $A$ is not an SV-ring.

The final example of this section shows that SV does not imply finite rank in a semiprime $f$-ring.

Example 3.7. Let $\mathbf{R}[[x]]$ denote the ring of formal power series over the real field, in one indeterminate. It is well known that this is a valuation domain. We totally order $\mathbf{R}[[x]]$ lexicographically, so that $1 \gg x \gg x^{2} \gg \cdots$. Note that under this ordering the elements which exceed 1 are invertible, because the multiplicative units of $\mathbf{R}[[x]]$ are precisely the series with nonzero constant term. Therefore, $\mathbf{R}[[x]]$ has bounded inversion. 
Now, let $\mathbf{R}_{0}[[x]]$ denote the ideal of series with 0 constant term. For each $n \in \mathbf{N}$, let $A_{n}=\mathbf{R}_{0}[[x]]$, and let $A$ be the direct sum of the $A_{n}$, ordered coordinatewise. Finally, let $B=\{(a, r): a \in A, r \in \mathbf{R}\}$. Define addition coordinatewise in $B$, and multiplication in the way that one does classically, so as to adjoin an identity to a ring; that is

$$
(a, r)(b, s)=(a b+r b+a s, r s) \text {. }
$$

Finally, partially order $B$ by declaring $(a, r)>0$ if $r>0$, or $r=0$ and $a>0$. Then it is easy to see that $B$ is a semiprime $f$-ring with identity. Furthermore, the reader may easily verify the following:

(i) $M=\{(a, 0): a \in A\}$ is the unique maximal ideal of $B$.

(ii) The minimal prime ideals of $B$ are: $P_{n}=\left\{(a, 0) \in B: a_{n}=0\right\}$ for each $n \in \mathbf{N}$. Moreover, for each $n, B / P_{n}=\mathbf{R}[[x]]$.

(iii) $B$ is an SV-algebra with bounded inversion, having infinite rank.

\section{UNIFORMLY COMPLETE SV-ALGEBRA}

Suppose that $A$ is an $f$-ring with identity. A sequence $\left\{a_{n}\right\}$ in an abelian $\ell$-group is said to be uniformly Cauchy if for each positive integer $p$ there is a positive integer $N(p)$ so that $n, m \geq N(p)$ implies that $p\left|a_{n}-a_{m}\right| \leq 1$. We say that $\left\{a_{n}\right\}$ converges uniformly to $a$ if for each positive integer $p$ there is a positive integer $N(p)$ so that $n \geq N(p)$ implies that $p\left|a_{n}-a\right| \leq 1$. The $f$-ring $A$ is uniformly complete if every uniformly Cauchy sequence converges uniformly to a unique limit. (Note: As pointed out in [HdP1], this version of the definition of uniform completeness implies that the ring is also archimedean.)

It is known that every uniformly complete $f$-algebra with identity has bounded inversion and square roots of positive elements (see [HdP1, Theorems 3.4 and 3.9]).

Now here is the principal theorem in this article.

Theorem 4.1. Every uniformly complete SV-algebra with identity has finite rank. Proof. We first show that every maximal ideal has finite rank. Then, as a theorem of independent interest, we show that if every maximal ideal has finite rank, then the rank of the algebra is finite.

Suppose that $A$ is a uniformly complete SV-algebra with identity. Suppose, by way of contradiction, that the maximal ideal $M$ contains the distinct minimal primes $P_{1}, P_{2}, \ldots, P_{n}, \ldots$ Viewing $\operatorname{Min}(A)$ as an infinite Hausdorff space we may suppose, without loss of generality that the points $P_{1}, P_{2}, \ldots$ form a discrete subspace in $\operatorname{Min}(A)$. Fix a free ultrafilter $U$ on $\mathbf{N}$. Now define the ideal $P$ as follows:

$$
f \in P \leftrightarrow\left\{n \in \mathbf{N}: f \in P_{n}\right\} \in U .
$$

It is shown in 4.8 of $[\mathrm{HJ}]$ that $P \in \operatorname{Min}(A)$, and it should be clear that $P \subset$ $M$. We now show that $A / P$ is not a valuation ring, which is the contradiction we desire.

Using the discreteness of the points $P_{n}$ in $\operatorname{Min}(A)$ we may select, for each $n \in \mathbf{N}$, positive $c_{n} \notin P_{n}$, such that each $c_{n} \leq 1$, and the $c_{n}$ are pairwise disjoint. Let

$$
b=\sum_{n \in \mathbf{N}} c_{n} / 2^{n} \text { and } a=\sum_{n \in \mathbf{N}} c_{n} /\left(n 2^{n}\right) .
$$


Obviously, $0<a \leq b$. Now, suppose that $x \in A$ such that $x b-a \in P$. Notice that $|x b-a|=\sum_{n \in \mathbf{N}}|x-(1 / n)| c_{n} / 2^{n}$, and $|x b-a| \in P_{n}$, for all $n$ belonging to a set $T \in U$. Since minimal prime ideals are convex, it follows that $|x-(1 / n)| c_{n} \in P_{n}$, for each $n \in T$. Since $c_{n} \notin P_{n}$ this means that $|x-(1 / n)| \in P_{n}$, and so $x \equiv 1 / n \bmod M$, infinitely often, which is evidently absurd. This proves that every maximal ideal of $A$ has finite rank.

Now let us state the second claim as a separate result.

Theorem 4.2. Suppose that $A$ is a uniformly complete $f$-algebra with identity, in which every maximal ideal has finite rank. Then $\operatorname{rk}(A)$ is finite.

Proof. Let us suppose again, by way of contradiction, that the ranks of the maximal ideals of $A$ are not bounded. Then we may select a discrete set of points $M_{1}, M_{2}, \ldots, M_{n}, \ldots \in \operatorname{Max}(A)$, so that the sequence $\left\{r_{n}=\operatorname{rk}\left(M_{n}, A\right)\right\}$ is strictly increasing. Let $M \in \operatorname{cl}\left\{M_{1}, M_{2}, \ldots\right\}$. Let $U$ be a free ultrafilter on $\mathbf{N}$, selected this time so that $U$ contains the traces of all neighborhoods of $M$ in $\operatorname{Max}(A)$ upon $\left\{M_{1}, M_{2}, \ldots\right\}$. For each $n \in \mathbf{N}$ let $\left\{P_{n}(i): 1 \leq i \leq r_{n}\right\}$ be the set of minimal prime ideals contained in $M_{n}$. Now, define the following set of ideals:

$$
f \in Q_{n} \leftrightarrow\left\{k \in \mathbf{N}: f \in P_{k}(n)\right\} \in U .
$$

(Note: Technically speaking, the preceding definition should be interpreted as $f \in Q_{n}$ if and only if $\left\{k \in \mathbf{N}: P_{k}(n)\right.$ is defined and $\left.f \in P_{k}(n)\right\} \in U$; however, for a given $n, P_{k}(n)$ is defined for all but a finite number of subscripts.)

Once again, each $Q_{n}$ is a minimal prime ideal. Since the ultrafilter $U$ extends the set of traces of neighborhoods of $M$ upon $\left\{M_{1}, M_{2}, \ldots\right\}$, each $Q_{n} \subseteq M$. To contradict the assumption that the ranks are finite, it suffices to show that the $Q_{n}$ are distinct. This is done in the following manner: as the $M_{n}$ form a discrete subspace of $\operatorname{Max}(A),\left\{P_{n}(k): n \in \mathbf{N}, 1 \leq k \leq r(n)\right\}$ also forms a discrete subspace of $\operatorname{Min}(A)$. Therefore, once again we may select a set of pairwise disjoint elements $x_{n}(k)$, such that $x_{n}(k) \notin P_{n}(k)$. If $m \neq k$, define $a$ and $b$ by $a=\sum_{n} x_{n}(k) / 2^{n}$ and $b=\sum_{n} x_{n}(m) / 2^{n}$. Then $a \wedge b=0$, and it should be clear that $a \notin Q_{k}$ while $b \notin Q_{m}$. Hence the $Q_{n}$ are distinct, violating the hypothesis of this theorem.

(Note: The proof of Theorem 4.1 is now also complete.)

In view of Proposition 3.1 we now have the following corollary.

Corollary 4.2.1. Let $X$ be a compact space. Then

(a) If $X$ is an $S V$-space then it has finite rank.

(b) If every point of $X$ has finite rank then $X$ has finite rank.

(c) If $X$ is an $S V$-space then there is a positive integer $k$ such that, for every $n>k$, any $n$ pairwise disjoint cozero sets have disjoint closures.

We do not know if every uniformly closed $f$-algebra with identity that has finite rank is an SV-ring. We call this latter assertion the converse of Theorem 4.1. By Proposition 3.2(2), $A$ has finite rank if and only if $A^{*}$ has finite rank, and it is shown in [HJo] that if $A=A^{*}$ is uniformly closed, then $A$ is $\ell$-isomorphic to $C(X)$, where $X=\operatorname{Max}(A)$ is the compact space of maximal ideals of $A$ in the hull-kernel topology. So, it would suffice to verify the converse 
of Theorem 4.1 by showing that every compact space of finite rank is an SVspace. Next, we capitalize on Theorem 2.14 to prove a special case of this desired result.

First, let us agree to call a positive element $x$ in a semiprime $f$-ring $A$ with identity and bounded inversion indecomposable at the maximal ideal $M$ if $x+O(M)$ is indecomposable in $A / O(M)$. For $C(X)$ the above definition translates as follows. A function $f \in C(X)$ is called indecomposable at the point $p \in \beta X$ if it is indecomposable at $M_{p}$. (This means that if $f=g+h$, with $g$ and $h$ disjoint, then one of the two vanishes on a neighborhood of $p$.)

Theorem 4.3. Suppose that $A$ is a uniformly complete $f$-algebra with identity. Then $A$ is an $S V$-algebra if and only if the following conditions hold:

(a) $A$ has finite rank, and

(b) for every maximal ideal $M$ and every pair $0<a \leq b$, with $b$ indecomposable at $M$, there is an $x \in A$ such that $a-x b \in O(M)$.

Proof. Suppose $A$ is an SV-algebra. Then (a) holds by Theorem 4.1. Theorem 2.11 implies $A / O(M)$ has a finite basis for each maximal ideal $M$. Note that $A / O(M)$ is local and inherits both the bounded inversion property and the presence of square roots of positive elements from $A$. If $0<a \leq b$, and $b$ is indecomposable at $M$, then $b+O(M)$ is special in $A / O(M)$. By Theorem 2.14 , the equation $a=x b$ can be solved modulo $O(M)$. So (b) holds.

Now suppose that (a) and (b) hold. Since $A$ has finite rank, each $A / O(M)$ has a finite basis by Theorem 2.11 . Theorem 2.14 and condition (b) say exactly that $A / O(M)$ is an SV-ring. Now, if $P$ is any minimal prime ideal of $A$, there is a maximal ideal $M$ (containing $P$ ) so that $P / O(M)$ is a minimal prime ideal of $A / O(M)$. Finally, $A / P=(A / O(M)) / P / O(M)$, which is a valuation ring, proving that $A$ is an SV-algebra.

Now we apply Theorem 4.3 to $C(X)$, with $X$ compact.

Theorem 4.4. Suppose that $X$ is a compact space. Then $X$ is an $S V$-space if and only if it has finite rank and for each $p \in X$ and each pair $0<f<g$, where $g$ is indecomposable at $p$, there is an $h$ in $C(X)$ such that $f=g h$ on a neighborhood of $p$.

A commutative ring $A$ with identity will be called locally Prüfer if each of its localizations is Prüfer, i.e., if $A / O(M)$ is a Prüfer ring for each $M \in \operatorname{Max}(A)$. As was noted in Lemma 2.17, a local commutative Prüfer ring with identity is quasi-Bezout; that is, finitely generated regular ideals are principal. It follows then from Proposition 2.15 that if $A$ is a semiprime $f$-ring with identity of finite rank that is closed under bounded inversion and locally Prüfer, then $A / O(M)$ is a valuation domain. In Theorem 1 of [MW], it is shown that in a semiprime $f$-ring that is closed under bounded inversion, if this latter holds for every $M \in$ $\operatorname{Max}(A)$, then $A$ is Bezout. Thus we have shown:

Theorem 4.5. Suppose that $A$ is a commutative semiprime $f$-ring with identity and the bounded inversion property. If $A$ has finite rank and is locally Prüfer, then $A$ is Bezout (and hence an SV-ring).

It is a fair question, whether Theorem 4.5 holds if "locally Prüfer" is replaced by "Prüfer". Topologically speaking: is every quasi-F space (defined in Section 5) of finite rank an F-space? A negative answer follows in the next section. 
Theorem 4.1 allows us to give another characterization of SV-rings for a $C(X)$. This characterization is analogous to the characterization of F-spaces given in 14.29 of [GJ].

Theorem 4.6. Let $X$ be a compact topological space. The following are equivalent.

1. $X$ is an $S V$-space.

2. For every $x \in X$, there is a $k \in \mathbf{N}$ such that for any cozero set $Y \subseteq X$ and $l \in C^{*}(Y)$ there are $l_{1}, l_{2}, \ldots, l_{k} \in C(X)$ and zero sets $Z_{1}, Z_{2}, \ldots, Z_{k}$ such that $\left.l_{i}\right|_{Z_{i} \cap Y}=\left.l\right|_{Z_{i} \cap Y}$ for each $i$ and $\bigcup Z_{i}$ is a neighborhood of $x$.

Proof. Suppose that $X$ is an SV-space. Let $x \in X$. By Theorem 4.1, there are only finitely many minimal prime ideals $P_{1}, P_{2}, \ldots, P_{k}$ that are contained in $M_{x}$. Let $Y=\operatorname{coz}(g)$ be a cozero set of $X$ and let $l \in C^{*}(Y)$. We may assume without loss of generality that $|l| \leq 1$. Define $k_{1}, k_{2} \in C(X)$ by

$$
k_{1}(x)= \begin{cases}0, & \text { if } x \in X \backslash Y \\ (l \vee 0)(x) g(x), & \text { if } x \in Y\end{cases}
$$

and

$$
k_{2}(x)= \begin{cases}0, & \text { if } x \in X \backslash Y \\ (-l \vee 0)(x) g(x), & \text { if } x \in Y .\end{cases}
$$

Since $l$ is bounded, $k_{1}, k_{2}$ are continuous on $X$. Then $0 \leq k_{1}, k_{2} \leq g$ and since $X$ is an SV-space, there exists for $i=1,2, \ldots, k$, elements $l_{1 i}, l_{2 i} \in$ $C(X)^{+}$such that $k_{1}-l_{1 i} g, k_{2}-l_{2 i} g \in P_{i}$. For each $i$, let $l_{i}=l_{1 i}-l_{2 i}$ and $Z_{i}=Z\left(l_{i}\right)$. Since $l=(l \vee 0)-(-l \vee 0)$, it follows that $\left.l_{i}\right|_{Z_{i} \cap Y}=\left.l\right|_{Z_{i} \cap Y}$. Now $\bigcup_{i=1}^{n} Z_{i}=Z\left(\prod_{i=1}^{n}\left(\left|k_{1}-l_{1 i} g\right|+\left|k_{2}-l_{2 i} g\right|\right)\right.$ and $\prod_{i=1}^{n}\left(\left|k_{1}-l_{1 i} g\right|+\left|k_{2}-l_{2 i} g\right|\right) \epsilon$ $\bigcap_{i=1}^{n} P_{i}=O\left(M_{x}\right)$. So $\bigcup_{i=1}^{n} Z_{i}$ must be a neighborhood of $x$ and (2) holds.

Now suppose that (2) holds. Let $P$ be a minimal prime ideal of $C(X)$. We will show that $C(X) / P$ is a valuation domain. Suppose $0 \leq f \leq g \bmod$ $P$. Then $0 \leq f \leq g+q$ for some $q \in P$. The ideal $P$ is contained in a unique maximal ideal $M_{x}$ for some $x \in X$. If $g+q \notin M_{x}$ then there is an $h \in O\left(M_{x}\right)$ such that $g+q+h$ is a multiplicative unit. Now define $k \in C(X)$ by $k=f /(g+q+h)$. Note that on $Z(h), f \equiv k(g+q)$. Therefore $f-k(g+q) \in O\left(M_{x}\right) \subseteq P$ and $f=k g \bmod P$. If $g+q \in M_{x}$, define $l \in C^{*}(\operatorname{coz}(g+q))$ by $l=f /(g+q)$. By hypothesis there are $l_{1}, l_{2}, \ldots, l_{k} \in$ $C(X)$ and $Z_{1}, Z_{2}, \ldots, Z_{k}$ such that for each $i,\left.l_{i}\right|_{Z_{i} \cap(\operatorname{coz}(g+q))}=\left.l\right|_{Z_{i} \cap(\operatorname{coz}(g+q))}$ and $\bigcup_{i=1}^{n} Z_{i}$ is a neighborhood of $x$. So $\prod_{i=1}^{n}\left(f-l_{i}(g+q)\right) \in O\left(M_{x}\right) \subseteq P$. Because $P$ is prime, $f-l_{i}(g+q) \in P$ for some $i$. So $f=l_{i} g \bmod P$.

We now turn to topological considerations.

\section{SV-SPACES AND SPACES OF FINITE RANK}

All spaces considered will be Tychonoff spaces. Recall that a subspace $Y$ of a space $X$ is said to be $C^{*}$-embedded in $X$ if every $f \in C^{*}(Y)$ has a continuous extension over $X$. If each dense cozero set of $X$ is $C^{*}$-embedded in $X$, then $X$ is said to be a quasi-F space; see [DHH, Section 5] where it is shown that $X$ is a quasi-F space if and only if $C(X)$ is quasi-Bezout. In what follows, we almost always confine our discussion to compact (Hausdorff) spaces because in studying SV-spaces, it suffices to consider those that are compact as was noted 
above and in [HW1]. In many instances, more general results are known for noncompact spaces.

A continuous surjection $f: X \rightarrow Y$ is said to be irreducible if no proper closed subset of $X$ is mapped by $f$ onto $Y$. It is shown in [DHH] that if $X$ is compact, there is an essentially unique quasi-F space $Q F(X)$ that maps irreducibly onto $X$ minimally in the sense that any continuous surjection of a compact quasi-F space factors through $Q F(X)$; for details see [DHH] or [HVW]. The space $Q F(X)$ is called the quasi-F cover of $X$.

A subspace is called a regular closed set if it is the closure of its interior. Let $Z(X)$ denote the family of zero sets of functions in $C(X)$, and let $Z^{\#}(X)$ denote the set of regular closed members of $Z(X)$. Then $Z^{\#}(X)$ is a lattice and it is shown in [HVW, 2.11-2.12] that for any compact space $X$, the continuous surjection $\varphi: Q F(X) \rightarrow X$ induces a lattice isomorphism of $Z^{\#}(Q F(X))$ onto $Z^{\#}(X)$. Thus, by Proposition 2.2 of [CM], the map $\varphi^{\prime}: C(X) \rightarrow C(Q F(X))$ such that $\varphi^{\prime}(f)=f \circ \varphi^{\prime}$ for each $f \in C(X)$ is a rigid embedding. So, by Proposition 3.2(1), we have:

Proposition 5.1. If a compact space has finite rank, then so does its quasi-F cover.

The space $[0,1]$ has infinite rank at each of its points while its quasi-F cover is extremally disconnected and hence has rank 1. (See [DHH, Theorem 4.7].) So, the converse of this proposition fails. We do not know if the analogue of 5.1 holds for SV-spaces. In particular, we do not know if every quasi-F space of finite rank is an SV-space.

It is shown in [HdP2, 6.2] that $Q F(X)$ is an F-space if and only if:

(*) If $C_{1}, C_{2}$ are disjoint cozero sets, then there are zero sets $Z_{1}, Z_{2}$ such that $C_{1} \subseteq Z_{1}, C_{2} \subseteq Z_{2}$, and $\operatorname{int}\left(Z_{1} \cap Z_{2}\right)=\varnothing$.

Thus, $(*)$ is a sufficient but not necessary condition for $Q F(X)$ to be an SV-space.

As was shown in [HJ] and [HM], $\operatorname{Min}(C(X))$ is compact and basically disconnected if and only if the lattice $\mathrm{P}_{r}(C(X))$ of principal polars in $C(X)$ is a Boolean algebra. See Section 2. Recall that a space is basically disconnected if closures of cozero sets are open. Clearly every basically disconnected space is an F-space.

Next, we give the previously advertised example of a ring that is Prüfer but not locally Prüfer. It appears in [MW] for slightly different purposes. We repeat it for the sake of completeness while leaving the verification of some details to the reader.

Example 5.2. Let $X$ denote the space obtained by attaching the free union of two copies of the compact space $\beta \omega \backslash \omega$ at a non-P-point $q$. It is well known that $\beta \omega \backslash \omega$ is an F-space in which every zero set is a regular closed set, and it follows that $X$ is a quasi-F space because it has no proper dense cozero set. Thus $C(X)$ is a Prüfer ring. Because it is the union of two closed F-spaces, it is an SV-space as was shown in [HW1]. Hence $C(X)$ has finite rank by Theorem 4.1. If it were also locally Prüfer, then it would be Bezout by Theorem 4.5. This cannot be the case because there are two minimal prime ideals of $C(X)$ contained in the maximal ideal $M_{q}$.

In view of Proposition 5.1, it seems natural to ask if the inverse image of a compact space of finite rank under an irreducible continuous surjection must 
have finite rank. We shall answer this question in the negative. In what follows, we abbreviate $\operatorname{Min}(C(X))$ by $m X$, and assume that it carries the hull-kernel topology. A nonempty closed subspace $E$ of a space $X$ is called a P-set if $E \subseteq Z(f)$ for any $f \in C(X)$ implies $E \subseteq \operatorname{Int} Z(f)$.

Example 5.3. Let $X$ be a compact F-space. One can then view $m X$ as a topological space whose underlying set is $X$ and which has $\left\{\operatorname{cl}_{X} C: C\right.$ is a cozero set of $X\}$ as an open base. (See [DHKV] for a discussion of this.) Obviously the identity map from $m X$ onto $X$ is a continuous surjection. (This corresponds to mapping the unique minimal prime ideal of $X$ that "lives at" the point $x$ of $X$ to the unique maximal ideal $M_{x}$ of $C(X)$ consisting of members of $C(X)$ that vanish at $x$.) Let $j$ denote this identity map and $\beta j$ its extension to $\beta(m X)$. Clearly $\beta j$ is a perfect continuous surjection. Suppose that it were not irreducible. Then there would be a regular closed proper subset $A$ of $\beta(m X)$ for which $\beta j(A)=X$. As $A$ is regular closed in $\beta(m X), A \cap m X$ is dense in $A$ and so $\beta j(A \cap m X)$ is dense in $X$. But $\beta j(A \cap m X)=A \cap X$, so $A \cap X$ is dense in $X$. But $A \cap m X$ is a proper regular closed subset of $m X$, so there is a nonempty cozero set $C$ of $X$ such that $\operatorname{cl}_{X} C \subseteq m X \backslash(A \cap m X)=X \backslash(A \cap X)$, contradicting the fact that $A \cap X$ is dense in $X$. Consequently we conclude that $\beta j$ is indeed irreducible.

There is in [DHKV, Corollary 6] an example in ZFC of a compact F-space $X$ in which every zero set is regular closed and which contains a compact P-set $E$, and a continuous surjection $g: X \rightarrow[0,1]$ which maps $E$ irreducibly onto $[0,1]$. Suppose now that $X$ is a space with these specifications.

It is shown in the proof of Theorem 3 [DHKV] that if $M$ is a countable dense subset of $[0,1]$, and $V(M)=\bigcup\left\{\right.$ int $\left._{X} g^{-1}(p): p \in M\right\}$, then $V(M) \in$ $\operatorname{coz}(m X)$ and $E \subseteq \operatorname{cl}_{m X} V(M)$. It should be clear that if $M$ and $S$ are disjoint countable dense subsets of the interval $[0,1]$, then $V(M) \cap V(S)=\varnothing$. Thus, if $\{M(i): i<\omega\}$ is a countable family of pairwise disjoint countable dense subsets of $[0,1]$, and we let $W(i)(i<\omega)$ be pairwise disjoint cozero sets of $\beta(m X)$, for which $V(M(i))=W(i) \cap m X$, we obtain an infinite pairwise disjoint family of cozero sets of $\beta(m X)$ for which the $\beta(m X)$-closures all contain $E$. By Proposition 3.1, all points of $E$ have infinite rank in $\beta(m X)$.

Thus, $\beta j$ maps the space $\beta(m X)$ of infinite rank onto the F-space $X$ in which each point has rank 1 .

For our next result we need some additional background information.

A topological space $X$ is called weakly Lindelöf if each open cover of $X$ contains a countable subfamily, the union of which is dense in $X$. If every family of pairwise disjoint open sets is countable we say that $X$ satisfies the $c c c$. It is noted in [PW, 3P] that if $X$ satisfies the $c c c$ then every open subspace of $X$ is weakly Lindelöf.

Suppose that $Y$ is a subspace of $X$. Then $Y$ is $z$-embedded in $X$ if every cozero set of $Y$ is the trace on $Y$ of a cozero set of $X$. We say that $Y$ is

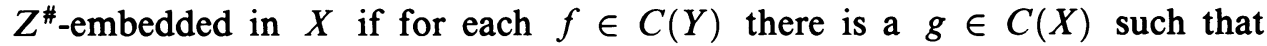
$\operatorname{cl}_{X}\left(\right.$ int $\left._{X}(Z(g))\right) \cap Y=\operatorname{cl}_{Y}\left(\right.$ int $\left._{Y}(Z(f))\right)$. When $Y$ is dense in $X$, then the restriction map induces an embedding of $C(X)$ as an $f$-subalgebra of $C(Y)$; then it is easy to check that $Y$ is $Z^{\#}$-embedded in $X$ precisely when $C(X)$ is rigidly embedded in $C(Y)$.

It is shown in [HVW, 3.2], that a $C^{*}$-embedding is always a $z$-embedding, 
which in turn is always a $Z^{\#}$-embedding. If $Y$ is a dense subspace of $X$ which is $Z^{\#}$-embedded, and $X$ is quasi-F, then $Y$ is $C^{*}$-embedded.

Finally, in 3.7 of [HVW] it is shown that every dense weakly Lindelöf subspace and every cozero set is $Z^{\#}$-embedded.

Applying Proposition 3.2(1) and Corollary 1.8.1, we get:

Proposition 5.4. Suppose $X$ is a compact space.

(a) If $X$ has finite rank, then so does every cozeroset and every dense weakly Lindelöf subspace.

(b) If $X$ is both an $S V$-space and a quasi-F space, then so is every dense cozero set and every dense weakly Lindelöf subspace.

Recall also that weakly Lindelöf and cozero subspaces of F-spaces are Fspaces as is noted in [HVW]. From this it is easy to show:

Proposition 5.5. If a compact space is finitely an F-space, then so is any weakly Lindelöf subspace.

Our next result shows that every infinite compact space of finite rank contains a plethora of copies of $\beta \omega$.

Proposition 5.6. Every infinite compact space of finite rank contains a copy of $\beta \omega$.

Proof. Suppose that $X$ is an infinite compact space of finite rank. Let $m$ denote the largest positive integer such that the set $E=E_{m}$ of points of rank > $m$ is finite. Then there is a countably infinite discrete subset $S=\left\{x_{1}, x_{2}, \ldots\right\}$ of points of rank $m$, the closure of which is disjoint from $E$. We claim that

$$
\text { if } y \in \operatorname{cl}(S) \backslash S \text {, then } S \text { is } C^{*} C^{*} \text {-embedded in } S \cup\{y\} \text {. }
$$

For, otherwise, there is a partition $S=V_{1} \cup V_{2}$ into infinite subsets, so that both $V_{1}$ and $V_{2}$ contain $y$ in their closures. Let $T_{1}$ and $T_{2}$ be distinct free ultrafilters on $\omega$ such that $\left\{i<\omega: x_{i} \in V_{i}\right\} \in T_{k}$ for $k=1,2$. For each $i<\omega$, let $\left\{P_{i j}: 1 \leq j \leq m\right\}$ denote the set of minimal prime ideals contained in $M_{x_{i}}$. Next, let $P_{j}\left(T_{k}\right)=\left\{f \in C(X):\left\{i<\omega: f \in P_{i j}\right\} \in T_{k}\right\}$ for $k=1,2$ and $1 \leq j \leq m$. As in the proofs of Theorems 4.1 and 4.2, each $P_{j}\left(T_{k}\right)$ is a minimal prime ideal contained in $M_{y}$, thus making the rank of $y$ at least $2 m$, which is a contradiction. This establishes $(*)$.

This implies that $\operatorname{cl}(S)$ is homeomorphic to $\beta S=\beta \omega$, and the proof is complete.

Owing to Corollary 1.8 .1 , the preceding result gives the following corollary, a property which (compact) SV-spaces share with F-spaces; see 14N.5 of [GJ].

Corollary 5.7. Every infinite closed subspace of a compact space of finite rank has at least $2^{c}$ points.

As usual, let $Z(X)$ denote the lattice of zero sets of the space $X$ and $\operatorname{coz}(X)$ denote the lattice of cozero sets of $X$. If $S \subseteq Z(X)$, we use $c S$ to denote $\{X \backslash Z: Z \in S\}$. Evidently, $c S$ is a family of cozero sets. An ultrafilter of subsets of $\operatorname{coz}(X)$ is called a cozero-ultrafilter on $X$. The following is an unpublished result of J. Vermeer; the proof is somewhat tedious, but straightforward, and is therefore not included. 
Proposition 5.8. The mapping $P \rightarrow c\{Z(f): f \in P\}$ is a bijection between the set $\operatorname{Min}(C(X))$ and the set of cozero-ultrafilters on $X$.

The preceding proposition allows us to present a topological characterization of SV-spaces.

Recall that a totally ordered integral domain $A$ is real-closed if (i) every positive element of $A$ has a square root, (ii) every polynomial of odd degree over $A$ has a root in $A$, and (iii) $A$ is a valuation domain with bounded inversion. (See [CD] and [HW1] for more information; it is shown in [CD] that the real-closed domains are the ones which are convex in their real-closed field of quotients.)

A prime $z$-ideal $P$ of $C(X)$ is called real-closed if $C(X) / P$ is a real-closed domain. (An ideal $J$ of $C(X)$ is a $z$-ideal if $f \in J$ and $Z(g)=Z(f)$ imply that $g \in J$.)

Theorem 5.9. Let $X$ be a (Tychonoff) space. Then $X$ is an $S V$-space if and only if the following condition is satisfied: if $V$ is a cozero set, $U$ a cozero-ultrafilter on $X$, and $f \in C^{*}(V)$ then there exists $C \in \operatorname{coz}(X) \backslash U$ such that $\left.f\right|_{V \backslash C}$ can be extended continuously to $X$.

Proof. It is known-see 14.7 of [GJ]-that every minimal prime ideal is a $z$-ideal. In 2.2 of [HW1] it is shown that $X$ is an SV-space if and only if every minimal prime ideal is real-closed. Further, 2.4 of [HW1] shows that if $P$ is a prime $z$-ideal of $C(X)$, then $P$ is real-closed if and only if for each cozero set $V$ of $X$ and each $f \in C^{*}(X)$ there is a $w \in P$ so that $\left.f\right|_{V \cap Z(w)}$ extends continuously to all of $X$. Combining all this with Proposition 5.8, and translating appropriately, we obtain the stated claim.

One of the puzzling and even frustrating aspects of the study of spaces of finite rank is the inability to make conclusions about the rank of a space from bounds placed on the ranks of points out of a dense subset. For example, if $X$ is any space, it would be reasonable to suppose that $\operatorname{rk}(\beta X)=\sup \{\operatorname{rk}(X, p)$ : $p \in X\}$. This is not so in general, although we can say the following.

Theorem 5.10. If $X$ is a normal space, then $\operatorname{rk}(\beta X)=\sup \{\operatorname{rk}(X, p): p \in X\}$. Proof. First, since $C(\beta X)=C^{*}(X)$, the bounded subring of $C(X)$, and is rigidly embedded in $C(X)$, these two $f$-rings have the same spaces of maximal ideals and also the same spaces of minimal prime ideals; (see the comments leading up to Proposition 3.2, as well as those preceding Proposition 1.9). So, $\operatorname{rk}(X, p)=\operatorname{rk}(\beta X, p)$, for each $p \in X$.

Now, suppose that $\operatorname{rk}(\beta X, q) \geq k$, for some point $q \in \beta X$. This implies that there is a family $\{V(i): 1 \leq i \leq k\}$ of pairwise disjoint cozero sets of $\beta X$ so that their $\beta X$-closures all contain $q$. Next, since

$$
\mathrm{cl}_{\beta X} V(i)=\operatorname{cl}_{\beta X}\left(\mathrm{cl}_{X}(V(i) \cap X)\right),
$$

we have that

$$
\begin{aligned}
\bigcap\left\{\operatorname{cl}_{\beta X} V(i): 1 \leq i \leq k\right\} & =\bigcap\left\{\operatorname{cl}_{\beta X}\left(\operatorname{cl}_{X}(V(i) \cap X)\right): 1 \leq i \leq k\right\} \\
& =\operatorname{cl}_{\beta X}\left(\bigcap\left\{\operatorname{cl}_{X}(V(i) \cap X): 1 \leq i \leq k\right\}\right),
\end{aligned}
$$

the latter identity owing to the assumption that $X$ is normal. 
Thus, $\bigcap\left\{\operatorname{cl}_{X}(V(i) \cap X): 1 \leq i \leq k\right\} \neq \varnothing$; on the other hand, the $V(i) \cap X$ are $k$ pairwise disjoint cozero sets of $X$. This says that any point of $X$ lying in all their closures has rank at least $k$. This completes the proof of the theorem.

The conclusion of Theorem 5.10 need not hold if the hypothesis of normality is dropped. A (Tychonoff) space $X$ is called an $F^{\prime}$-space if disjoint cozero sets have disjoint closures. Equivalently, $X$ is an $F^{\prime}$-space if and only if each of its points has rank 1. Every normal $F^{\prime}$-space is an F-space, and in Example 8.14 of [GH] an example is given of an $\mathrm{F}^{\prime}$-space $Y$ that is not an F-space. If $W$ is an $\mathrm{F}^{\prime}$-space that is not an F-space then $\beta W$ must have a point of rank greater than 1; for otherwise $\beta W$ and hence $W$ would be an F-space. In [D, Example 1.10], A. Dow gives an example of a locally compact $F^{\prime}$-space $T$ that is not an F-space such that $\beta T=T \cup\{\infty\}$ is the union of two closed F-spaces, each containing $\infty$. Thus $\sup \{\operatorname{rk}(T, p): p \in T\}=1<\operatorname{rk}(\beta T)=2$. (The interested reader may verify that for the space $Y$ in Example 8.14 of [GH], $\beta Y$ contains a point of infinite rank.)

In the next proposition we show that a certain class of points always have rank 1.

Suppose that $X$ is a Tychonoff space; a nonisolated point $p \in \beta X$ is remote if it does not lie in the $\beta X$-closure of any closed subset of $X$ which is nowhere dense. Note that, necessarily, a remote point lies in $\beta X \backslash X$. For more information on these points, such as an account of sufficient conditions for their existence and number, see [PW, 4AH]. For instance, 4AH(10) concludes that if $X$ is not pseudo-compact, but has a countable $\pi$-base, then there are $\exp \left(\exp \left(\aleph_{0}\right)\right)$ remote points. (A $\pi$-base is a family of open sets with the feature that every open set contains a member of the family.) The results presented in $4 \mathrm{AH}$ of $[\mathrm{PW}]$ originally appeared in [CS] and [vD].

Proposition 5.11. Suppose that $X$ is a normal space; then every remote point of $\beta X$ has rank 1.

Proof. Suppose, by way of contradiction, that $p$ is remote and $p$ belongs to the $\beta X$-closures of both $V$ and $W$, where $V$ and $W$ are disjoint cozero sets of $\beta X$. As $X$ is normal, $p$ lies in the $\beta X$-closure of $\operatorname{cl}_{X}(V \cap X) \cap \operatorname{cl}_{X}(W \cap X)$, which is a closed, nowhere dense subset of $X$. This is a contradiction, and hence $p$ must have rank 1 .

Example 5.12. Applying the proposition to $\beta \mathbf{Q}$, which according to [VD] has a dense set $E$ of remote points, we see that $\beta \mathbf{Q}$ has a dense subset of points of rank 1. However, since $\mathbf{Q}$ is a metric space, it is easy to find, for any closed, nowhere dense subset $C \subseteq \mathbf{Q}$ an infinite family of pairwise disjoint cozerosets of $\mathbf{Q}$, all of which contain $C$ in their closures. Now, any nonremote point of $\beta \mathbf{Q}$ lies in the $\beta \mathbf{Q}$-closure of some such $C$; clearly then, every nonremote point of $\beta \mathbf{Q}$ has infinite rank. Since these points include the rational numbers themselves, we have a space $(\boldsymbol{\beta} \mathbf{Q})$ which is partitioned into two dense subsets: in one all the points have rank 1 ; in the other all the points have infinite rank.

The next two results look at what happens to the ranks of points in product spaces. First, some local information. Recall, from Corollary 1.8.2 and the comment following Proposition 3.1, that if $Y$ is a closed subspace of the compact space $X$, and $p \in \operatorname{int}_{X} Y$, then the rank of $p$ is the same whether one 
computes it in $X$ or $Y$. This observation makes the following fairly transparent.

Proposition 5.13. Suppose that $X$ and $Y$ are compact spaces, and $p$ and $q$ are points of $X$ and $Y$ respectively. Then

(a) $\operatorname{rk}(X \times Y,(p, q)) \geq \operatorname{rk}(X, p) \operatorname{rk}(Y, q)$.

(b) If $p$ is isolated, then $\operatorname{rk}(X \times Y,(p, q))=\operatorname{rk}(Y, q)$.

Sketch of Proof. (a) is an immediate consequence of 3.1. As to (b), simply note that an isolated point has rank 1 , and that $\{p\} \times Y$ is clopen in the product.

The estimate in part (a) in the preceding proposition can be strict; in fact, even the result of $(b)$ does not hold in general for points of rank 1 . The next theorem takes care of that. We have not been able to determine, however, whether (b) holds if $p$ is a $P$-point of $X$.

Before stating the theorem in question, we need a lemma.

Lemma 5.14. $\beta \omega \times \beta \omega$ has infinite rank.

Proof. Let $g: \beta(\omega \times \omega) \rightarrow \beta \omega \times \beta \omega$ be the Stone extension of the identity map on $\omega \times \omega$. By 16.11 of [CN], there exists a $p \in \beta \omega \backslash \omega$ for which $\left|g^{-1}\{(p, p)\}\right|=\exp \left(\exp \left(\aleph_{0}\right)\right)$. Let $\{d(i): i<\omega\}$ be a countably infinite discrete subset of $g^{-1}\{(p, p)\}$, and $\{A(i): i<\omega\}$ be a family of pairwise disjoint, infinite subsets of $\omega \times \omega$, so that the $\beta(\omega \times \omega)$-closure of $A(i)$ contains $d(i)$. Then it is clear that $(p, p)$ lies in the $(\beta \omega) \times \beta \omega)$-closure of each $A(i)$, and so the rank of $(p, p)$ is infinite.

Theorem 5.15. If $X$ and $Y$ are infinite, compact $F$-spaces, then $X \times Y$ has infinite rank.

Proof. By Proposition 5.6, both spaces contain a copy of $\beta \omega$, and so $X \times Y$ contains a (closed) copy of $\beta \omega \times \beta \omega$. Now apply Corollary 1.8.1.

We close the section with two results; the first for compact finitely F-spaces, where the set of points of rank 1 is dense. Like Proposition 5.6, the other shows that in a compact space of finite rank there are many closed subspaces which are F-spaces. We leave the details of the proof of the latter to the reader.

Proposition 5.16. If $X$ is compact and finitely an F-space, then $X$ has an open and dense subset of points of rank 1.

Proof. Suppose $X=\bigcup\{X(i): 1 \leq i \leq n\}$, where each $X(i)$ is a compact F-space. Let $T=\bigcup\left\{\operatorname{bd}_{X} X(i): 1 \leq i \leq n\right\}$, where $\operatorname{bd}_{X} K$ stands for the topological boundary of $K$. Then $T$ is a closed nowhere dense subset of $X$, and $X \backslash T$ is a locally compact, dense subspace. Since each point of $X \backslash T$ lies in the interior of a closed F-subspace, its rank is 1 .

Proposition 5.17. Suppose that $X$ is a compact space of rank $k<\infty$. If $\{V(i)$ : $1 \leq i \leq k\}$ is a family of $k$ pairwise disjoint cozerosets of $X$ then $\bigcup\{\mathrm{cl}(V(i))$ : $1 \leq i \leq k\}$ is an F-space.

\section{ANOTHER CHARACTERIZATION OF SV-ALGEBRAS AND UNSOLVED PROBLEMS}

An ideal $I$ of an $f$-ring $A$ is said to be saturated if $a+b \in I$ and $a b=0$ imply $a$ and $b$ are in $I$. Because $a=(a \vee 0)-(-a \vee 0),|a|=(a \vee 0)+(-a \vee 0)$, and $(a \vee 0)(-a \vee 0)=0$, it is clear that: 
(甘) If $I$ is saturated and $a \in A$, then $a \in I$ if and only if $|a| \in I$.

It is easy to see that every $\ell$-ideal $J$ of $A$ is saturated. For, if $a=b+c \in J$ and $b c=0$, then $|a|=|b|+|c|$, so $|b| \leq|a| \in J$. So $b$ is in the $\ell$-ideal $J$. Similarly, $c \in J$. On the other hand, in any totally ordered ring, every ideal is saturated. So the converse of the latter need not hold in general. We can, however, show that:

Theorem 6.1. Every saturated ideal of a uniformly closed SV-algebra $A$ with identity is an $\ell$-ideal.

Proof. Recall that every uniformly closed $f$-ring is commutative and semiprime. If $I$ is an ideal of $A$, and $M$ is a maximal ideal of $A$, let $I^{*}(M)=I^{*}=$ $(I+O(M)) / O(M)$. We show next that:

$(\ddagger)$ If $I$ is a saturated ideal of $A$, then $I^{*}$ is saturated in $A / O(M)$.

To see this, suppose $(x+O(M))+(y+O(M)) \in I^{*}$ and $x y \in O(M)$ for some $x, y \in A$. Then $x+y=z+c$ for some $z \in I$ and $c \in O(M)$. If $y^{\prime}=y-c$, then $x+y^{\prime}=z \in I$ and $x y^{\prime} \in O(M)$. By definition of $O(M)$, there is an $a \in A^{+} \backslash M$ such that $x y^{\prime} a=0$. Since $A$ is uniformly closed, there is a $b>0$ such that $b^{2}=a$. Then $x b+y^{\prime} b=z b \in I$, and $(x b)\left(y^{\prime} b\right)=0$. Because $I$ is saturated, $x b$ and $y^{\prime} b$ are in $I$. Hence $x b$ and $y b$ are in $I+O(M)$. Finally, because $b$ is invertible $\bmod O(M)$, both $x+O(M)$ and $y+O(M)$ are in $I^{*}$. So $(\ddagger)$ holds.

Next, we show that $I^{*}$ is an $\ell$-ideal. To do this, it suffices by $(\sharp)$ to show that for $x, y \in A / O(M), x \in I^{*}$ whenever $0<x \leq y$ and $y \in I^{*}$. Suppose the hypothesis of the latter holds. By Theorem 2.5, $y=\sum_{i=1}^{k} y_{i}$, where $\left\{y_{1}, \ldots, y_{k}\right\}$ is a set of pairwise disjoint positive special elements each of which is in the saturated ideal $I^{*}$. Then $x=\sum_{i=1}^{k} x \wedge y_{i}$. Since $x \wedge y_{i} \leq y_{i}$ and $y_{i}$ is special, it follows from Theorem 2.14 that there is an $a_{i}$ such that $x \wedge y_{i}=a_{i} y_{i}$ for $1 \leq i \leq k$. Hence $x \in I^{*}$ and we know that this ideal is an $\ell$-ideal.

We will show finally that $I$ is an $\ell$-ideal. Because $A$ is uniformly closed, its space of maximal ideals $\operatorname{Max}(A)$ is compact; see [HJo]. Suppose $0<x \leq y \in I$. Then $x+O(M) \leq y+O(M) \in I^{*}(M)$, so by the above, $x+O(M) \in I^{*}(M)$. Thus, there is a $y_{M} \in I$ with $\left(x-y_{M}\right) \in O(M)$, and hence there is a positive $a_{M} \notin M$ such that $\left(x-y_{M}\right) a_{M}=0$. Let $h^{c}\left(a_{M}\right)=\left\{K \in \operatorname{Max}(A): a_{M} \notin K\right\}$. Clearly $\left\{h^{c}\left(a_{M}\right): M \in \operatorname{Max}(A)\right\}$ is an open cover of the compact space $\operatorname{Max}(A)$. So there is a positive integer $k$ and maximal ideals $M_{1}, \ldots, M_{k}$ such that $a=a_{M_{1}}+\cdots+a_{M_{k}}$ is invertible and $x a_{M_{i}}=y_{M_{i}} a_{M_{i}}$ for $1 \leq i \leq k$. It follows that $x=a^{-1} \sum_{i=1}^{k} y_{M_{i}} a_{M_{i}} \in I$. By $(\sharp)$, this completes the proof of the theorem.

Recall that an ideal $Q$ of an $f$-ring is called pseudoprime if $a b=0$ implies $a$ or $b$ is in $Q$. Clearly, every pseudoprime ideal is saturated. In [HL], it is shown that a commutative semiprime $f$-ring with bounded inversion is an SV-ring if and only every pseudoprime ideal is an $\ell$-ideal. Hence we have:

Corollary 6.2. A uniformly closed $f$-algebra is an $S V$-algebra if and only if each of its saturated ideals is an $\ell$-ideal.

Recall from 14.25 of [GJ] that $X$ is an F-space if and only if every ideal of $C(X)$ is an $\ell$-ideal. 
The major problems we have been unable to solve follow. Suppose $A$ is a uniformly closed $f$-algebra with identity.

Problem 6.3. If $A$ has finite rank, must it be an SV-algebra? It was noted in Section 5 that we can assume without loss of generality that $A=A^{*}=C(X)$ for some compact space $X$.

Problem 6.4. If $A=C(X)$ is an SV-algebra, and $X$ is compact, must $X$ be a finite union of compact F-spaces?

As noted in Section 5, an affirmative answer to this question would show that every compact SV-space $X$ has a dense set of points of rank 1 and hence is a compactification of an $\mathrm{F}^{\prime}$-space.

On the other hand, we do not know the answer to:

Problem 6.5. If $A$ is a SV-algebra, must it have a point of rank 1 ?

We conclude with a special case of Problem 6.3.

Problem 6.6. Must the quasi-F cover $Q F(X)$ of a compact SV-space $X$ be an SV-space? Recall from Proposition 5.1 that under this assumption, $Q F(X)$ has finite rank.

We are unable to answer this latter question even if $X$ is finitely an F-space.

$$
\text { AdDED JUly 7, } 1993
$$

Recently, A. Dow and R. Levy have shown (in unpublished work) that if $\mathrm{CH}$ holds, then any compact space of finite rank and weight $2^{\omega}$ has a dense set of points of rank 1 .

\section{REFERENCES}

[AF] M. Anderson and T. Feil, Lattice-ordered groups, Reidel Texts Math. Sci., Kluwer, Dordrecht, 1988.

[BKW] A. Bigard, K. Keimel, and S. Wolfenstein, Groupes et anneaux réticulés, Lecture Notes in Math., vol. 608, Springer-Verlag, Berlin, Heidelberg, New York, 1977.

[CD] G. Cherlin and M. Dickmann, Real closed rings. I, Fund. Math. 126 (1986), 147-183.

[CM] P. Conrad and J. Martinez, Complemented lattice-ordered groups, Indag. Math. (N.S.) 1 (1990), 281-297.

[CN] W. Comfort and S. Negrepontis, The theory of ultrafilters, Springer-Verlag, Berlin, Heidelberg, New York, 1974.

[CS] S. B. Chae and J. H. Smith, Remote points and G-spaces, Topology Appl. 11 (1980), 243-246.

[D] A. Dow, F-spaces and $F^{\prime}$-spaces, Pacific J. Math. 108 (1983), 275-283.

[vD] E. van Douwen, Remote points, Dissertationes Math. (Rozprawy Mat.) 188 (1981), $1-45$.

[DHH] F. Dashiell, A. W. Hager, and M. Henriksen, Order-Cauchy completions of rings and vector lattices of continuous functions, Canad. J. Math. 32 (1980), 657-685.

[DHKV] A. Dow, M. Henriksen, R. Kopperman, and J. Vermeer, The space of minimal prime ideals of a $C(X)$ need not be basically disconnected, Proc. Amer. Math. Soc. 104 (1988), 317-320.

[G] R. Gilmer, Multiplicative ideal theory, Marcel Dekker, New York, 1972.

[GH] L. Gillman and M. Henriksen, Rings of continuous functions in which every finitely generated ideal is principal, Trans. Amer. Math. Soc. 82 (1956), 366-391. 
[GJ] L. Gillman and M. Jerison, Rings of continuous functions, Graduate Texts in Math., 43, Springer-Verlag, Berlin, Heidelberg, New York, 1976.

[GIH] A. M. W. Glass and W. C. Holland (Editor), Lattice-ordered groups, Kluwer, Dordrecht and Boston, 1989.

[H] M. Henriksen, Semiprime ideals of f-rings, Sympos. Math. 21 (1977), 401-407.

[HJ] M. Henriksen and M. Jerison, The space of minimal prime ideals of a commutative ring, Trans. Amer. Math. Soc. 115 (1965), 110-130.

[HJo] M. Henriksen and D. Johnson, On the structure of a class of archimedean lattice ordered algebras, Fund. Math. 50 (1961), 73-93.

[HK] M. Henriksen and R. Kopperman, A general theory of structure spaces with applications to spaces of prime ideals, Algebra Universalis 28 (1991), 349-376.

[HL] M. Henriksen and S. Larson, Semiprime f-rings that are subdirect products of valuation domains, Ordered Algebraic Structures, The 1991 Conrad Conference, Kluwer Academic, Dordrecht, 1993.

[HM] A. W. Hager and J. Martinez, Fraction-dense algebras and spaces, Canad. J. Math. 45 (1993), 977-996.

[HdP1] C. B. Huijsmans and B. de Pagter, Ideal theory in f-algebras, Trans. Amer. Math. Soc. 269 (1982), 225-245.

[HdP2] _ Maximal d-ideals in a Riesz space, Canad. J. Math. 35 (1983), 1010-1029.

[HR] A. Hager and L. Robertson, Representing and ringifying a Riesz space, Sympos. Math. 31 (1977), 411-431.

[HS] M. Henriksen and F. A. Smith, Sums of z-ideals and semiprime ideals, General Topology Rel. Modern Anal. Algebra 5 (1982), 272-278.

[HVW] M. Henriksen, J. Vermeer, and R. G. Woods, Quasi-F covers of Tychonoff spaces; Trans. Amer. Math. Soc. 303 (1987), 779-803.

[HW1] M. Henriksen and R. Wilson, When is $C(X) / P$ a valuation ring for every prime ideal $P$ ?, Topology and Appl. 44 (1992), 175-180.

[HW2] _ Almost discrete SV-spaces, Topology Appl. 46 (1992), 89-97.

[K] K. Koh, On functional representation of a ring without nilpotent elements; Canad. Math. Bull. 14 (1971), 349-352.

[LZ] W. Luxemburg and Zaanen, Riesz spaces. I, North-Holland, Amsterdam, 1971.

[MW] J. Martinez and S. Woodward, Bezout and Prüfer f-rings; Comm. Algebra (to appear).

[PW] J. Porter and R. G. Woods, Extensions and absolutes of Hausdorff spaces, SpringerVerlag, Berlin, Heidelberg New York, 1989.

[S] F. Sik, Zur Theorie der halbgeordneten Gruppen, Czechoslovak. Math. J. 10 (1960), 400-424.

[Su] H. Subramanian, $\ell$-prime ideals in f-rings, Bull. Soc. Math. France 95 (1967), 193-203.

[TN] N. Thakare and S. Nimbhorkar, Spaces of minimal prime ideals of a ring without nilpotent elements, J. Pure Appl. Algebra 27 (1983), 75-85.

[W] S. Woodward, On f-rings that are rich in idempotents, Univ. of Florida dissertation, 1992.

Department of Mathematics, Harvey Mudd College, Claremont, California 91711

E-mail address: henriksen@hmc.edu

Department of Mathematics, Loyola Marymount University, Los Angeles, CaliforNIA 90045

E-mail address: slarson@lmumail.1mu.edu

Department of Mathematics, University of Florida, Gainesville Florida 32611

E-mail address: martinez@math.ufl.edu

Department of Mathematics, University of Manitoba, Winnipeg, Manitoba R3T 2N2, CANADA

E-mail address: rgwoods@ccm. umanitoba.ca 